\title{
THE GLOBAL WELL-POSEDNESS AND SCATTERING FOR THE 5D DEFOCUSING CONFORMAL INVARIANT NLW WITH RADIAL INITIAL DATA IN A CRITICAL BESOV SPACE
}

\author{
CHANGXING MIAO, JIANWEI YANG, AND TENGFEI ZHAO
}

\begin{abstract}
In this paper, we obtain the global well-posedness and scattering for the radial solution to the defocusing conformal invariant nonlinear wave equation with initial data in the critical Besov space $\dot{B}_{1,1}^{3} \times \dot{B}_{1,1}^{2}\left(\mathbb{R}^{5}\right)$. This is the five dimensional analogue of [6], which is the first result on the global well-posedness and scattering of the energy subcritical nonlinear wave equation without the uniform boundedness assumption on the critical Sobolev norms employed as a substitute of the missing conservation law with respect to the scaling invariance of the equation. The proof is based on exploiting the structure of the radial solution, developing the Strichartz-type estimates and incorporation of the strategy in [6], where we also avoid a logarithm-type loss by employing the inhomogeneous Strichartz estimates.
\end{abstract}

\section{INTRODUCTION}

We consider the solutions $u$ to

$$
\begin{cases}\partial_{t t} u-\Delta u+\mu|u|^{p} u=0, & (t, x) \in \mathbb{R} \times \mathbb{R}^{d}, \\ \left(u(0), \partial_{t} u(0)\right)=\left(u_{0}, u_{1}\right), & x \in \mathbb{R}^{d},\end{cases}
$$

where $\mu= \pm 1, d \geq 1$, and $p>0$. If $\mu=1$ the equation (1.1) is described as defocusing, otherwise focusing. There is a natural scaling symmetry for (1.1), i.e., if we let $u_{\lambda}(t, x)=\lambda^{\frac{2}{p}} u(\lambda t, \lambda x)$ for $\lambda>0$, then $u_{\lambda}$ is also a solution to the equation (1.1) with initial data $\left(\lambda^{\frac{2}{p}} u_{0}(\lambda x), \lambda^{\frac{2}{p}+1} u_{1}(\lambda x)\right)$ preserving the $\dot{H}^{s_{p}} \times \dot{H}^{s_{p}-1}\left(\mathbb{R}^{d}\right)$ norm of the initial data, where we define the critial regulairity as $s_{p}=\frac{d}{2}-\frac{2}{p}$. At least, the solutions to (1.1) formally conserve the energy

$$
E\left(u(t), \partial_{t} u(t)\right):=\frac{1}{2} \int_{\mathbb{R}^{5}}\left|\nabla_{x} u(t)\right|^{2} d x+\frac{1}{2} \int_{\mathbb{R}^{5}}\left|\partial_{t} u(t)\right|^{2} d x+\frac{\mu}{p+2} \int_{\mathbb{R}^{5}}|u(t)|^{p+2} d x,
$$

which is also invariant under the scaling if $s_{p}=1$. In view of this, we say the Cauchy problem (1.1) is energy critical when $s_{p}=1$, subcritical for $s_{p}<1$ and supercritical when $s_{p}>1$.

Lindblad and Sogge [19] proved the local theory of the Cauchy problem (1.1) in the minimal regularity spaces. In fact, if $d \geq 2$ and $p \geq \frac{d+3}{d-1}$, the Cauchy problem (1.1) with initial data in the critical spaces $\dot{H}^{s_{p}} \times \dot{H}^{s_{p}-1}\left(\mathbb{R}^{d}\right)$ is locally well-posed. The global theory for the Cauchy problem (1.1) with $\mu=1$ and $s_{p} \leq 1$ has been studied extensively. While for the focusing case, even the solution with smooth initial data may blow up at finite time. For more related results, we refer the reader to [26] and the references therein.

In this paper, we will consider global existence and scattering of the solutions to (1.1). In general, a solution $u$ is said to be scattering, if it is a global solution and approaches a linear solution as $t \rightarrow \pm \infty$. In the cases of $d \geq 2$ and $p \geq \frac{d+3}{d-1}$, the solution to (1.1) with small initial datum in the critical Sobolev spaces is globally well-posed and scattering, see [19.

2000 Mathematics Subject Classification. Primary: 35B40, 35Q40.

Key words and phrases. Nonlinear wave equation, Strichartz estimates, Scattering, hyperbolic coordinates, Morawetz esimates. 
For the defocusing energy critical wave equation (1.1), Grillakis [10] first established the global existence theory for classical solution when $d=3$. The results for other dimensions are proved in [11, 25]. scattering results for large energy data are proved in [1, 2, 22] by establishing variants of the Morawetz estimates (see Morawetz [21])

$$
\iint_{\mathbb{R}^{1+d}} \frac{|u|^{\frac{2 d}{d-2}}}{|x|} d x d t \leq C_{d} E\left(u_{0}, u_{1}\right)
$$

where $C_{d}$ is a constant depending on $d$. While in focusing energy critical cases, the Morawetz estimates (1.3) fails. And the scattering results do not hold in general, since (1.1) has a ground state $W(x)=$ $\left(1+\frac{|x|^{2}}{d(d-2)}\right)^{-\frac{d-2}{2}}$. In the cases of $3 \leq d \leq 5$, Kenig-Merle [15] proved the scattering result for solution with initial data such that $E\left(u_{0}, u_{1}\right)<E(W, 0)$ and $\left\|u_{0}\right\|_{\dot{H}^{1}\left(\mathbb{R}^{d}\right)}<\|W\|_{\dot{H}^{1}\left(\mathbb{R}^{d}\right)}$. In their proofs, the main ingredient is the concentration compactness/rigidity theorem method introduced by [14]. This method is powerful and plays an important role in study of many other nonlinear dispersive equations. We refer to [16, 18, 13, and the references therein.

For the defocusing subcritical equation (1.1), the global existence has been proved for solution with initial data in the energy space $\dot{H}^{1} \times L^{2}\left(\mathbb{R}^{d}\right)$ by Ginibre-Velo [7, 8]. However, there are no scattering results even for solutions with initial datum in $\left(\dot{H}^{1} \cap \dot{H}^{s_{p}}\right) \times\left(L^{2} \cap \dot{H}^{s_{p}-1}\right)\left(\mathbb{R}^{d}\right)$.

Recently, Dodson [6] proved scattering results for the defocusing cubic wave equation with the initial datum belonging to the space $\dot{B}_{1,1}^{2} \times \dot{B}_{1,1}^{1}\left(\mathbb{R}^{3}\right)$, which is a subspace of $\dot{H}^{1 / 2} \times \dot{H}^{-1 / 2}\left(\mathbb{R}^{3}\right)$. We remark that this is the first work that gives scattering result for large data in the critical Sobolev space without any a priori bound on the critical norm of the solution. Dodson's strategy consists of three steps:

(1) By establishing some new Strichartz-type estimates, one can show that the solution is in the energy space $\dot{H}^{1} \times L^{2}\left(\mathbb{R}^{3}\right)$ up to some free evolutions. Then this decomposition enables one to prove the global well-posedness of the solution.

(2) To obtain the scattering result, a conformal transformation is applied to show that the energy part of the solution has finite energy in hyperbolic coordinates. Then from the conformal invariance of the equation and a Morawetz-type inequality, one can deduce that $\|u\|_{L_{t, x}^{4}\left(\mathbb{R} \times \mathbb{R}^{5}\right)} \leq$ $C\left(\left\|\left(u_{0}, u_{1}\right)\right\|_{\dot{B}_{1,1}^{3} \times \dot{B}_{1,1}^{2}\left(\mathbb{R}^{5}\right)}, \delta_{1}\right)$, where the parameter $\delta_{1}$ relies on the scaling and spatial profiles of the initial data.

(3) Finally, one can remove the dependence of $\delta_{1}$ by employing the profile decomposition, which completes the proof.

Let $S(t)(f, g)$ be the solution of Cauchy problem to the free wave equation

$$
\begin{cases}\partial_{t t} v-\Delta v=0, & (t, x) \in \mathbb{R} \times \mathbb{R}^{5}, \\ \left.\left(v, \partial_{t} v\right)\right|_{t=0}=(f(x), g(x)), & x \in \mathbb{R}^{5} .\end{cases}
$$

For the sake of statement, we introduce the following notation as

$$
\dot{S}(t)(f, g) \triangleq \partial_{t} S(t)(f, g), \text { and } \vec{S}(t)(f, g) \triangleq(S(t)(f, g), \dot{S}(t)(f, g)) .
$$

We consider the Cauchy problem of nonlinear wave equation

$$
\left\{\begin{array}{l}
\partial_{t t} u-\Delta u+|u| u=0, \quad(t, x) \in \mathbb{R} \times \mathbb{R}^{d}, \\
\left(u(0), \partial_{t} u(0)\right)=\left(u_{0}, u_{1}\right), \quad x \in \mathbb{R}^{d},
\end{array}\right.
$$

Our main result can be stated as: 
Theorem 1. For any radial initial data $\left(u_{0}, u_{1}\right) \in \dot{B}_{1,1}^{3} \times \dot{B}_{1,1}^{2}\left(\mathbb{R}^{5}\right)$, the solution $u$ to (1.5) is globally well-posed and scattering, i.e., there exists $\left(u_{0}^{ \pm}, u_{1}^{ \pm}\right) \in \dot{H}^{1 / 2} \times \dot{H}^{-1 / 2}\left(\mathbb{R}^{5}\right)$ such that

$$
\lim _{t \rightarrow \pm \infty}\left\|\left(u(t), \partial_{t} u(t)\right)-\vec{S}(t)\left(u_{0}^{ \pm}, u_{1}^{ \pm}\right)\right\|_{\dot{H}^{\frac{1}{2}} \times \dot{H}^{-\frac{1}{2}}\left(\mathbb{R}^{5}\right)} \rightarrow 0 .
$$

Furthermore, there is a function $A:[0, \infty) \rightarrow[0, \infty)$, such that

$$
\|u\|_{L_{t, x}^{3}\left(\mathbb{R} \times \mathbb{R}^{5}\right)} \leq A\left(\left\|\left(u_{0}, u_{1}\right)\right\|_{\dot{B}_{1,1}^{3} \times \dot{B}_{1,1}^{2}\left(\mathbb{R}^{5}\right)}\right) .
$$

Remark 1.1. 1. This theorem extends the results of [6] to the 5-dimension case. Though the proof will utilize the strategy given in [6], but it is highly nontrivial.

2. Unlike the $3 D$ case, the dispersive estimate(see (2.20) ) gives a decay in time of order -2, which may cause a logarithmic failure when one estimates

$$
\|u\|_{L_{t}^{\frac{4}{3}} L_{x}^{4}\left(J \times \mathbb{R}^{5}\right)}+\sup _{t \in J}\left(t^{\frac{3}{4}}\|u\|_{L_{x}^{4}\left(\mathbb{R}^{5}\right)}\right),
$$

where $0 \in J$ is a local time interval. We circumvent this difficulty by using the inhomogenous Strichartz estimates in [27] and prove the global well-posedness of $u$.

3. For the scattering result, by reductions, we need to bound the $L_{t, x}^{3}$ of $w$ on the light cone $\{|x| \leq$ $\left.t+\frac{1}{2}\right\}$. We will define the hyperbolic energy by rewriting the equation (1.5) as the form

$$
\partial_{t t}\left(r^{2} w\right)-\partial_{r r}\left(r^{2} w\right)=-2 w-r^{2}|w| w .
$$

Observing that the additional term $2 w$ and the nonlinear term $r^{2}|w| w$ enjoy same sign, we can bound the $L_{t, x}^{3}$ norm of $w$ by applying a Morawetz-type inequality, if we assume the hyperbolic energy of $w$ is bounded.

4. To certifying the above assumption, we will make full use of the formula (2.19) for radial solution and the sharp Hardy inequality. In contrast to the $3 D$ case, some terms in formula (2.19) seem more difficult to dealt with. However, the integration domains of these terms are symmetric about the radius $r$, which also consists with the Huygens principle. This fact allows us apply the Hardy-Littlewood maximal functions to verifying the assumption.

Now, we give the outline of the proof. By the Strichartz estimates and a standard fix point argument, for initial data $\left(u_{0}, u_{1}\right)$, there exists a maximal time interval $I \subset \mathbb{R}$ such that there exists a unique solution $u$ (see Definition 2 in Section 2) to the equation (1.5) on $I \times \mathbb{R}^{5}$. We consider the global well-posedness by developing some Strichartz-type estimates (3.30). Utilizing the standard blowup criterion, we can show the global well-posedness of $u$.

Next, we claim the following proposition.

Proposition 1.2. For every radial initial data $\left(u_{0}, u_{1}\right) \in \dot{B}_{1,1}^{3} \times \dot{B}_{1,1}^{2}\left(\mathbb{R}^{5}\right)$, let $u$ be the corresponding solution to (1.5). Then there exists a parameter $\delta_{1}$ depending the initial data $\left(u_{0}, u_{1}\right)$ and a function $A:[0, \infty)^{2} \rightarrow[0, \infty)$ such that

$$
\|u\|_{L_{t, x}^{3}\left(\mathbb{R} \times \mathbb{R}^{5}\right)} \leq A\left(\left\|\left(u_{0}, u_{1}\right)\right\|_{\dot{B}_{1,1}^{3} \times \dot{B}_{1,1}^{2}\left(\mathbb{R}^{5}\right)}, \delta_{1}\right) .
$$

We prove Theorem 1 by employing this and establishing Proposition 4.1, where the proof provides an alternate proof of Lemma 6.2 in [6].

Finally, we need to prove Proposition 1.2. From the partition $u=v+w$, it suffices to show the boundedness of $L_{t, x}^{3}$ norm of $w$. We prove the hyperbolic energy of $w$ is uniformly bounded. Then, a Morawetz-type inequality yields that the $L_{t, x}^{3}$ norm of $w$ is bounded in the cone, which finishes the proof. 
This paper is organized as follows: Section 2 gives some tools from harmonic analysis and basic properties for the wave equation. And, in Section 3 we give the decomposition of $u$ and prove its global well-posedness. The existence of the function $A$ in (1.7) is shown in Section 4 based on the Proposition 1.2. Finally, in Section 5, we complete the proof by showing Proposition 1.2.

We end the introduction with some notations used throughout this paper. We use $\mathcal{S}\left(\mathbb{R}^{d}\right)$ to denote the space of Schwartz functions on $\mathbb{R}^{d}$. For $1 \leq p \leq \infty$, we define $L^{p}\left(\mathbb{R}^{d}\right)$ by the spaces of Lebesgue measurable functions with finite $L^{p}\left(\mathbb{R}^{d}\right)$-norm, which is defined by

$$
\|f\|_{L^{p}\left(\mathbb{R}^{d}\right)}=\left(\int_{\mathbb{R}^{d}}|f(x)|^{p} d x\right)^{1 / p}, \text { for } 1 \leq p<\infty,
$$

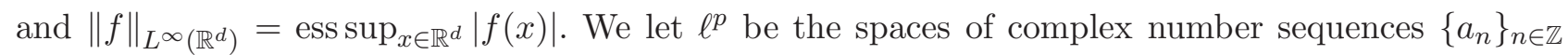
such that $\left\{a_{n}\right\}_{n \in \mathbb{Z}} \in l^{p}$ if and only if

$$
\left\|\left\{a_{n}\right\}\right\|_{\ell_{n}^{p}(\mathbb{Z})} \triangleq\left(\sum_{n}\left|a_{n}\right|^{p}\right)^{1 / p}<\infty, \text { for } 1 \leq p<\infty
$$

and $\left\|\left\{a_{n}\right\}\right\|_{\ell_{n}^{\infty}(\mathbb{Z})}:=\sup _{n}\left|a_{n}\right|<\infty$. We use $X \lesssim Y$ to mean that there exists a constant $C>1$ such that $X \leq C Y$, where the dependence of $C$ on the parameters will be clear from the context. We use $X \sim Y$ to denote $X \lesssim Y$ and $Y \lesssim X . A \ll B$ denotes there is a sufficiently large number $C$ such that $A \leq C^{-1} B$.

\section{BASIC TOOLS AND SOME ELEMENTARY PROPERTIES FOR THE WAVE EQUATION}

In this section, we recall some tools from harmonic analysis and useful results for the wave equation.

2.1. Some tools from harmonic analysis. Recall the Fourier transform of $f \in \mathcal{S}\left(\mathbb{R}^{d}\right)$ is defined by

$$
\widehat{f}(\xi)=(2 \pi)^{-d / 2} \int_{\mathbb{R}^{d}} f(x) e^{-i x \xi} d x
$$

which can be extended to Schwartz distributions naturally. We will make frequent use of the LittlewoodPaley projection operators. Specifically, we let $\varphi$ be a radial smooth function supported on the ball $|\xi| \leq 2$ and equal to 1 on the ball $|\xi| \leq 1$. For $j \in \mathbb{Z}$, we define the Littlewood-Paley projection operators by

$$
\begin{aligned}
\widehat{P_{\leq j} f}(\xi) & :=\varphi\left(\xi / 2^{j}\right) \widehat{f}(\xi), \\
\widehat{P_{>j} f}(\xi) & :=\left(1-\varphi\left(\xi / 2^{j}\right)\right) \widehat{f}(\xi), \\
\widehat{P_{j} f}(\xi) & :=\left(\varphi\left(\xi / 2^{j}\right)-\varphi\left(\xi / 2^{j-1}\right)\right) \widehat{f}(\xi) .
\end{aligned}
$$

The Littlewood-Paley operators commute with derivative operators and are bounded on the general Sobolev spaces. These operators also obey the following standard Bernstein estimates:

Lemma 2.1 (Bernstein estimates). For $1 \leq r \leq q \leq \infty$ and $s \geq 0$,

$$
\begin{aligned}
\left\||\nabla|^{ \pm s} P_{j} f\right\|_{L_{x}^{r}\left(\mathbb{R}^{d}\right)} & \sim 2^{ \pm j s}\left\|P_{j} f\right\|_{L_{x}^{r}\left(\mathbb{R}^{d}\right)}, \\
\left\||\nabla|^{s} P_{\leq j} f\right\|_{L_{x}^{r}\left(\mathbb{R}^{d}\right)} & \lesssim 2^{j s}\left\|P_{\leq j} f\right\|_{L_{x}^{r}\left(\mathbb{R}^{d}\right)} \\
\left\|P_{>j} f\right\|_{L_{x}^{r}\left(\mathbb{R}^{d}\right)} & \lesssim 2^{-j s}\left\||\nabla|^{s} P_{>j} f\right\|_{L_{x}^{r}\left(\mathbb{R}^{d}\right)} \\
\left\|P_{\leq j} f\right\|_{L^{q}\left(\mathbb{R}^{d}\right)} & \lesssim 2^{\left(\frac{d}{r}-\frac{d}{q}\right) j}\left\|P_{\leq j} f\right\|_{L_{x}^{r}\left(\mathbb{R}^{d}\right)}
\end{aligned}
$$

where the fractional derivative operator $|\nabla|^{\sigma}$ is defined by $\widehat{|\nabla|^{\sigma} f}(\xi)=|\xi|^{\sigma} \widehat{f}(\xi)$, for $\sigma \in \mathbb{R}$. 
Definition 1 (Homogeneous Besov spaces). Let $s$ be a real number and $1 \leq p, r \leq \infty$. We denote the homogeneous Besov norm by

$$
\|f\|_{\dot{B}_{p, r}^{s}\left(\mathbb{R}^{d}\right)}:=\left\|\left\{2^{j s}\left\|P_{j} f\right\|_{L^{p}\left(\mathbb{R}^{d}\right)}\right\}\right\|_{\ell_{j}^{r}(\mathbb{Z})},
$$

for $f \in \mathcal{S}\left(\mathbb{R}^{d}\right)$. Then the Besov space $\dot{B}_{p, r}^{s}\left(\mathbb{R}^{d}\right)$ is the completion of the Schwartz function under this norm.

We shall give the following radial Sobolev-type inequalities, which are analogous to $3 D$ cases established in [6]. We denote radial derivative by $\partial_{r} f(x)=\left(\frac{x}{|x|} \cdot \nabla\right) f(x)$ for any function $f$ defined on $\mathbb{R}^{5}$.

Lemma 2.2 (Radial Sobolev-type inequalities in Besov spaces). For any radial function $f \in \mathcal{S}\left(\mathbb{R}^{5}\right)$, we have

$$
\left\||x|^{2} f\right\|_{L^{\infty}\left(\mathbb{R}^{5}\right)} \lesssim\|f\|_{\dot{B}_{2,1}^{\frac{1}{2}}\left(\mathbb{R}^{5}\right)}
$$

Let $\left(u_{0}, u_{1}\right) \in \dot{B}_{1,1}^{3} \times \dot{B}_{1,1}^{2}\left(\mathbb{R}^{5}\right)$ be a radial function, then we have

$$
\begin{aligned}
\left\|\frac{1}{|x|^{2}} \partial_{r} u_{0}(x)\right\|_{L_{x}^{1}\left(\mathbb{R}^{5}\right)} & \left\|\frac{1}{|x|} \partial_{r r} u_{0}(x)\right\|_{L_{x}^{1}\left(\mathbb{R}^{5}\right)}+\left\|\frac{1}{|x|^{3}} u_{0}(x)\right\|_{L_{x}^{1}\left(\mathbb{R}^{5}\right)}+\left\||x|^{3} \partial_{r} u_{0}\right\|_{L_{x}^{\infty}\left(\mathbb{R}^{5}\right)} \lesssim\left\|u_{0}\right\|_{\dot{B}_{1,1}^{3}\left(\mathbb{R}^{5}\right)}, \\
& \left\|\frac{1}{|x|} \partial_{r} u_{1}(x)\right\|_{L_{x}^{1}\left(\mathbb{R}^{5}\right)}+\left\|\frac{1}{|x|^{2}} u_{1}(x)\right\|_{L_{x}^{1}\left(\mathbb{R}^{5}\right)}+\left\||x|^{3} u_{1}(x)\right\|_{L_{x}^{\infty}\left(\mathbb{R}^{5}\right)} \lesssim\left\|u_{1}\right\|_{\dot{B}_{1,1}^{2}\left(\mathbb{R}^{5}\right)} .
\end{aligned}
$$

Proof. We first consider (2.2). Since $f$ is radial, using polar coordinates, we have

$$
\begin{aligned}
P_{j} f(|x|) & =P_{j} f(x)=\int_{\mathbb{R}^{5}} \widehat{P_{j} f}(\xi) e^{i x \xi} d \xi \\
& =\int_{0}^{\infty} \int_{\mathbb{S}^{4}} \widehat{P_{j} f}(r) r^{4} e^{i r x \omega} d \sigma(\omega) d r .
\end{aligned}
$$

Recall the decay estimates of Fourier transform of the surface measure on the sphere

$$
\widehat{d \sigma_{\mathbb{S}^{4}}}(\xi) \leq C(1+|\xi|)^{-2}
$$

which and Hölder's inequality yield

$$
\left|P_{j} f(|x|)\right| \lesssim \int_{0}^{\infty}\left|\widehat{P_{j} f}(r)\right| r^{2}|x|^{-2} d r \lesssim|x|^{-2} 2^{\frac{1}{2} j}\left\|P_{j} f\right\|_{L^{2}}
$$

Then the inequality (2.2) follows from (2.6) and the definition of the Besov space.

Next, we consider (2.3) and (2.4). By the density of Schwartz functions in $\dot{B}_{1,1}^{3} \times \dot{B}_{1,1}^{2}\left(\mathbb{R}^{5}\right)$, we may assume that $u_{0}, u_{1} \in \mathcal{S}\left(\mathbb{R}^{5}\right)$. We claim that it suffices to show

$$
\begin{aligned}
\left\|\frac{1}{|x|^{2}} \partial_{r} u_{0}(x)\right\|_{L_{x}^{1}\left(\mathbb{R}^{5}\right)}+ & \left\|\frac{1}{|x|}\left|\Delta u_{0}(x)\right|\right\|_{L_{x}^{1}\left(\mathbb{R}^{5}\right)}+\left\|\frac{1}{|x|^{3}} u_{0}(x)\right\|_{L_{x}^{1}\left(\mathbb{R}^{5}\right)} \lesssim\left\|u_{0}\right\|_{\dot{B}_{1,1}^{3}\left(\mathbb{R}^{5}\right)}, \\
& \left\|\frac{1}{|x|} \partial_{r} u_{1}(x)\right\|_{L_{x}^{1}\left(\mathbb{R}^{5}\right)}+\left\|\frac{1}{|x|^{2}} u_{1}(x)\right\|_{L_{x}^{1}\left(\mathbb{R}^{5}\right)} \lesssim\left\|u_{1}\right\|_{\dot{B}_{1,1}^{2}\left(\mathbb{R}^{5}\right)} .
\end{aligned}
$$


To see this, by using the fact $\Delta f=\partial_{r r} f+\frac{4}{r} \partial_{r} f$ for radial function $f(x)$ on $\mathbb{R}^{5}$, we have

$$
\left\|\frac{1}{|x|} \partial_{r r} u_{0}(x)\right\|_{L_{x}^{1}\left(\mathbb{R}^{5}\right)} \lesssim\left\|\frac{1}{|x|^{2}} \partial_{r} u_{0}(x)\right\|_{L_{x}^{1}\left(\mathbb{R}^{5}\right)}+\left\|\frac{1}{|x|}\left|\Delta u_{0}(x)\right|\right\|_{L_{x}^{1}\left(\mathbb{R}^{5}\right)} \lesssim\left\|u_{0}\right\|_{\dot{B}_{1,1}^{3}\left(\mathbb{R}^{5}\right)}
$$

From the fundamental theorem of calculus and polar coordinates, for $y \in \mathbb{R}^{5} \backslash\{0\}$, we have

$$
|y|^{3}\left|\partial_{r} u_{0}(y)\right| \lesssim \int_{|y|}^{\infty} \int_{\mathbb{S}^{4}} r^{3}\left|\partial_{r r} u_{0}(r)\right| d \sigma(\omega) d r \lesssim\left\|\frac{1}{|x|} \partial_{r r} u_{0}(x)\right\|_{L_{x}^{1}\left(\mathbb{R}^{5}\right)} \lesssim\left\|u_{0}\right\|_{\dot{B}_{1,1}^{3}\left(\mathbb{R}^{5}\right)}
$$

and

$$
|y|^{3}\left|u_{1}(y)\right| \lesssim \int_{|y|}^{\infty} \int_{\mathbb{S}^{4}} r^{3}\left|\partial_{r r} u_{1}(r)\right| d \sigma(\omega) d r \lesssim\left\|\frac{1}{|x|} \partial_{r} u_{1}(x)\right\|_{L_{x}^{1}\left(\mathbb{R}^{5}\right)} \lesssim\left\|u_{1}\right\|_{\dot{B}_{1,1}^{2}\left(\mathbb{R}^{5}\right)}
$$

Hence, we are reduced to proving (2.7) and (2.8). We just give the estimate for the first term on the left hand side of (2.7), since others can be handled similarly. For $j \in \mathbb{Z}$, utilizing Bernstein's estimates and polar coordinates, we obtain

$$
\begin{aligned}
\left\|\frac{1}{|x|^{2}} \partial_{r} P_{j} u_{0}(x)\right\|_{L_{x}^{1}\left(\mathbb{R}^{5}\right)} & \lesssim \int_{0}^{\infty} \int_{\mathbb{S}^{4}} r^{2} \partial_{r} P_{j} u_{0}(r) d \sigma(\omega) d r \\
& \lesssim \int_{0}^{2^{-j}} \frac{1}{r^{2}}\left|\partial_{r}\left(P_{j} u_{0}\right)\right| r^{4} d r+\int_{2^{-j}}^{\infty} \frac{1}{r^{2}}\left|\partial_{r}\left(P_{j} u_{0}\right)\right| r^{4} d r \\
& \lesssim 2^{-3 j}\left\|\partial_{r}\left(P_{j} u_{0}\right)\right\|_{L_{r}^{\infty}\left(\mathbb{R}_{+}\right)}+2^{2 j}\left\|\partial_{r}\left(P_{j} u_{0}\right)\right\|_{L_{x}^{1}\left(\mathbb{R}^{5}\right)} \\
& \lesssim 2^{3 j}\left\|P_{j} u_{0}\right\|_{L_{x}^{1}\left(\mathbb{R}^{5}\right)}
\end{aligned}
$$

Thus, we have $\left\|\frac{1}{|x|^{2}} \partial_{r} u_{0}(x)\right\|_{L_{x}^{1}\left(\mathbb{R}^{5}\right)} \lesssim\left\|u_{0}\right\|_{\dot{B}_{1,1}^{3}\left(\mathbb{R}^{5}\right)}$.

As a direct consequence of Lemma 2.2, we have

$$
\left\||x|^{\frac{1}{2}} \partial_{r} u_{0}(x)\right\|_{L_{x}^{2}\left(\mathbb{R}^{5}\right)}+\left\||x|^{-\frac{1}{2}} u_{0}(x)\right\|_{L_{x}^{2}\left(\mathbb{R}^{5}\right)}+\left\||x|^{\frac{1}{2}} u_{1}(x)\right\|_{L_{x}^{2}\left(\mathbb{R}^{5}\right)} \lesssim\left\|\left(u_{0}, u_{1}\right)\right\|_{\dot{B}_{1,1}^{3} \times \dot{B}_{1,1}^{2}\left(\mathbb{R}^{5}\right)} .
$$

Lemma 2.3. Suppose $\chi(x) \in C_{c}^{\infty}\left(\mathbb{R}^{5}\right)$. Let $R=2^{k}$ be a dyadic number for $k \in \mathbb{Z}$ and denote $\chi_{R}(x)=\chi\left(\frac{x}{R}\right)$. Then we have

$$
\begin{aligned}
\left\|\chi_{R}(x) f\right\|_{\dot{B}_{2,1}^{\frac{1}{2}}\left(\mathbb{R}^{5}\right)} & \lesssim\|f\|_{\dot{B}_{2,1}^{\frac{1}{2}}\left(\mathbb{R}^{5}\right)}, \\
\left\|\chi_{R}(x) g\right\|_{\dot{B}_{2,1}^{-\frac{1}{2}}\left(\mathbb{R}^{5}\right)} & \lesssim\|g\|_{\dot{B}_{2,1}^{-\frac{1}{2}}\left(\mathbb{R}^{5}\right)},
\end{aligned}
$$

where the bound is independent of $R$. Furthermore, if $\chi(x)=1$ on $|x| \leq 1$, then for $(f, g) \in \dot{B}_{2,1}^{\frac{1}{2}} \times$ $\dot{B}_{2,1}^{-\frac{1}{2}}\left(\mathbb{R}^{5}\right)$, we have

$$
\lim _{R \rightarrow \infty}\left\|\left(1-\chi_{R}(x)\right) f\right\|_{\dot{B}_{2,1}^{\frac{1}{2}}\left(\mathbb{R}^{5}\right)}+\left\|\left(1-\chi_{R}(x)\right) g\right\|_{\dot{B}_{2,1}^{-\frac{1}{2}}\left(\mathbb{R}^{5}\right)}=0 .
$$

Proof. By scaling, to prove the inequalities (2.14) and (2.15), it suffices to prove the cases for $R=1$, which follows from a similar proof of Lemma 2.2 in [6]. On the other hand, (2.16) follows from (2.14), (2.15), and the fact that $C_{c}^{\infty} \times C_{c}^{\infty}\left(\mathbb{R}^{5}\right)$ is dense in $\dot{B}_{2,1}^{\frac{1}{2}} \times \dot{B}_{2,1}^{-\frac{1}{2}}\left(\mathbb{R}^{5}\right)$.

Finally, we need the following chain rule estimates for later use. 
Lemma 2.4 ( $C^{1}$-fractional chain rule, [4]). Suppose $G \in C^{1}(\mathbb{C}), s \in(0,1]$, and $1<q, q_{1}, q_{2}<\infty$ satisfying $\frac{1}{q}=\frac{1}{q_{1}}+\frac{1}{q_{2}}$. Then

$$
\left\||\nabla|^{s} G(u)\right\|_{L^{q}\left(\mathbb{R}^{d}\right)} \lesssim\left\|G^{\prime}(u)\right\|_{L^{q_{1}\left(\mathbb{R}^{d}\right)}}\left\||\nabla|^{s} u\right\|_{L^{q_{2}\left(\mathbb{R}^{d}\right)}} .
$$

2.2. Fundamental properties of the wave equations. Throughout the paper, by abuse of notations, we often write $u(t)=u(t, x)$ for simplicity and $u(t, r)=u(t, x)$ when $u(t, \cdot)$ is radially symmetric.

Recall the explicit formula for solution to the linear wave equation in 5 dimension,

$$
\begin{aligned}
S(t)(f, g)(x) & =\cos (t|\nabla|) f(x)+\frac{\sin (t|\nabla|)}{|\nabla|} g(x) \\
& =\frac{1}{3 \omega_{5}} \partial_{t}\left[\frac{1}{t} \partial_{t}\right]\left(t^{3} \int_{|y|=1} f(x+t y) d \sigma(y)\right)+\frac{1}{3 \omega_{5}} \frac{1}{t} \partial_{t}\left(t^{3} \int_{|y|=1} g(x+t y) d \sigma(y)\right),
\end{aligned}
$$

where $\omega_{5}$ is the surface area of the unit sphere in $\mathbb{R}^{5}$. When $(f, g)$ is radially symmetric, for $t>0$, (2.18) can be rewritten as

$$
\begin{aligned}
S(t)(f, g)(r)= & \frac{1}{2 r^{2}}\left[(r-t)^{2} f(r-t)+(r+t)^{2} f(r+t)\right]-\frac{t}{2 r^{3}} \int_{|r-t|}^{r+t} s f(s) d s \\
& +\frac{1}{4 r^{3}} \int_{|r-t|}^{r+t} s\left(s^{2}+r^{2}-t^{2}\right) g(s) d s .
\end{aligned}
$$

See also [23, 20, 5] for the radial solutions to general dimensions linear wave equation. From the explicit formula (2.18), we can obtain the following dispersive estimate.

Proposition 2.5 (Dispersive estimate).

$$
\left\|S(t)\left(u_{0}, u_{1}\right)\right\|_{L^{\infty}\left(\mathbb{R}^{5}\right)} \lesssim \frac{1}{t^{2}}\left[\left\|\nabla^{3} u_{0}\right\|_{L^{1}\left(\mathbb{R}^{5}\right)}+\left\|\nabla^{2} u_{1}\right\|_{L^{1}\left(\mathbb{R}^{5}\right)}\right] .
$$

Proof. We give the proof for completeness. Similar proof for $3 D$ case can be found in [17]. By (2.18), the free solution $S(t)\left(u_{0}, u_{1}\right)$ can be rewritten as

$$
\begin{aligned}
& \frac{1}{\omega_{5}} \int_{|y|=1} u_{0}(x+t y) d \sigma(y)+\frac{5 t}{3 \omega_{5}} \int_{|y|=1} y\left(\nabla u_{0}\right)(x+t y) d \sigma(y)+\frac{t^{2}}{3 \omega_{5}} \int_{|y|=1} y\left(\nabla^{2} u_{0}\right)(x+t y) y d \sigma(y) \\
& +\frac{t}{\omega_{5}} \int_{|y|=1} u_{1}(x+t y) d \sigma(y)+\frac{t^{2}}{3 \omega_{5}} \int_{|y|=1} y\left(\nabla u_{1}\right)(x+t y) d \sigma(y)
\end{aligned}
$$

which and the fundamental theorem of calculus yield (2.20). For instance, using polar coordinates, we can estimate the first term of (2.21) as

$$
\begin{aligned}
\left|\frac{1}{\omega_{5}} \int_{|y|=1} u_{0}(x+t y) d \sigma(y)\right| & =\left|\frac{1}{\omega_{5}} \int_{t}^{\infty} \int_{s}^{\infty} \int_{\tau}^{\infty} \int_{|y|=1} \frac{d^{3}}{d \rho^{3}}\left[u_{0}(x+\rho y)\right] d \sigma(y) d \rho d \tau d s\right| \\
& \lesssim \int_{t}^{\infty} \int_{s}^{\infty} \int_{\tau}^{\infty} \int_{|y|=1}\left|\nabla^{3} u_{0}\right|(x+\rho y) d \sigma(y) d \rho d \tau d s \\
& \lesssim \int_{t}^{\infty} \int_{s}^{\infty} \frac{1}{\tau^{4}} d \tau d s\left\|\nabla^{3} u_{0}\right\|_{L^{1}\left(\mathbb{R}^{5}\right)} \\
& \lesssim \frac{1}{t^{2}}\left\|\nabla^{3} u_{0}\right\|_{L^{1}\left(\mathbb{R}^{5}\right)} .
\end{aligned}
$$

And other terms can be dealt with similarly. 
We recall the Strichartz estimates of wave equation in $\mathbb{R}^{5}$. Let $I \subset \mathbb{R}$ be an interval. We denote the spacetime norm $L_{t}^{q} W_{x}^{s, r}\left(I \times \mathbb{R}^{5}\right)$ of a function $u(t, x)$ on $I \times \mathbb{R}^{5}$ by

$$
\|u\|_{L_{t}^{q} W_{x}^{s, r}\left(I \times \mathbb{R}^{5}\right)}:=\|\| u(t, x)\left\|_{W_{x}^{s, r}\left(\mathbb{R}^{5}\right)}\right\|_{L_{t}^{q}(I)}
$$

for $s \in \mathbb{R}, 1 \leq q, r \leq \infty$. We denote that a pair $(q, r)$ of exponents is admissible, if

$$
2 \leq q \leq \infty, 2 \leq r<\infty, \text { and } \frac{1}{q}+\frac{2}{r} \leq 1
$$

Moreover, we say $(q, r)$ is wave acceptable, provided

$$
1 \leq q<\infty, 2 \leq r \leq \infty, \frac{1}{q}<4\left(\frac{1}{2}-\frac{1}{r}\right),
$$

or $(q, r)=(\infty, 2)$.

Proposition 2.6 (Strichartz estimates [19, 9, 12]). Let $\left(u_{0}, u_{1}\right) \in \dot{H}^{1 / 2} \times \dot{H}^{-1 / 2}\left(\mathbb{R}^{5}\right)$ and $(q, r),(\tilde{q}, \tilde{r})$ be two admissible pairs. If $u$ is a weak solution to the wave equation $\partial_{t t} u-\Delta u=F(t, x)$ with initial data $\left(u_{0}, u_{1}\right)$, then we have

$$
\left\||\nabla|^{\rho} u\right\|_{L_{t}^{q} L_{x}^{r}\left(I \times \mathbb{R}^{5}\right)}+\sup _{t \in I}\left\|\left(u, u_{t}\right)\right\|_{\dot{H}^{1 / 2} \times \dot{H}^{-1 / 2}\left(\mathbb{R}^{5}\right)} \lesssim\left\|\left(u_{0}, u_{1}\right)\right\|_{\dot{H}^{1 / 2} \times \dot{H}^{-1 / 2}\left(\mathbb{R}^{5}\right)}+\left\||\nabla|^{-\mu} F\right\|_{L_{t}^{\tilde{q}^{\prime}} L^{\tilde{r}^{\prime}}\left(I \times \mathbb{R}^{5}\right)},
$$

provided that

$$
\rho=\frac{1}{q}+\frac{5}{r}-2 \text { and } \mu=\frac{1}{\tilde{q}}+\frac{5}{\tilde{r}}-2 .
$$

Proposition 2.7 (Inhomogeneous Strichartz estimates [27]). Suppose the exponent pairs $\left(q_{1}, r_{1}\right)$ and $\left(\tilde{q}_{1}, \tilde{r}_{1}\right)$ are wave acceptable, and satisfy the scaling condition

and the conditions

$$
\frac{1}{q}+\frac{1}{\tilde{q}}=2-2\left(\frac{1}{r_{1}}+\frac{1}{\tilde{r}_{1}}\right)
$$

Let $r \geq r_{1}, \tilde{r} \geq \tilde{r}_{1}, \rho \in \mathbb{R}$, and such that

$$
\frac{1}{q}+\frac{1}{\tilde{q}}<1, \quad \frac{1}{2} \leq \frac{\tilde{r}_{1}}{r_{1}} \leq 2
$$

$$
\rho+5\left(\frac{1}{2}-\frac{1}{r}\right)-\frac{1}{q}=1-\left(\tilde{\rho}+5\left(\frac{1}{2}-\frac{1}{\tilde{r}}\right)-\frac{1}{\tilde{q}}\right) .
$$

If $F(t, x) \in L_{t}^{\tilde{q}^{\prime}}\left(\mathbb{R} ; \dot{B}_{\tilde{r}^{\prime}, 2}^{-\tilde{\rho}}\left(\mathbb{R}^{5}\right)\right)$ and $u$ is a weak solution to the inhomogeneous wave equation

$$
-\partial_{t}^{2} u+\Delta u=F(t, x), \quad u(0)=u_{t}(0)=0,
$$

then

$$
\|u\|_{L_{t}^{q}\left(\mathbb{R} ; \dot{B}_{r, 2}^{\rho}\left(\mathbb{R}^{5}\right)\right)} \lesssim\|F(t, x)\|_{L_{t}^{\tilde{q}^{\prime}\left(\mathbb{R} ; \dot{B}_{\tilde{r}, 2}^{-\tilde{\rho}}\left(\mathbb{R}^{5}\right)\right)}}
$$

Next, we recall the well-posedness theory and the perturbation theory of the Cauchy problem (1.5).

Definition 2 (Solution). Let $I$ be a time interval such that $0 \in I$. We call function $u: I \times \mathbb{R}^{5} \rightarrow \mathbb{R}$ is a (strong) solution to the Cauchy problem (1.5) in $I$ if it satisfies $\left(u, u_{t}\right)(0)=\left(u_{0}, u_{1}\right)$,

$$
\left(u, u_{t}\right) \in C\left(I ; \dot{H}^{1 / 2} \times \dot{H}^{-1 / 2}\left(\mathbb{R}^{5}\right)\right) \cap L_{t, x}^{3}\left(I \times \mathbb{R}^{5}\right),
$$

and the integral equation

$$
u(t)=S(t)\left(u_{0}, u_{1}\right)-\int_{0}^{t} S(t-\tau)(0,|u| u(\tau)) d \tau
$$


for all $t \in I$.

Theorem 2 (Local well-posedness [19, 3]). Let $\left(u_{0}, u_{1}\right) \in \dot{H}^{1 / 2} \times \dot{H}^{-1 / 2}\left(\mathbb{R}^{5}\right)$ with

$$
\left\|\left(u_{0}, u_{1}\right)\right\|_{\dot{H}^{1 / 2} \times \dot{H}^{-1 / 2}\left(\mathbb{R}^{5}\right)} \leq A .
$$

There exists $\delta=\delta(A)>0$ such that, if

$$
\left\|S(t)\left(u_{0}, u_{1}\right)\right\|_{L_{t, x}^{3}\left([0, T] \times \mathbb{R}^{5}\right)} \leq \delta, \text { for some } T>0,
$$

then there exists a unique solution $u$ to (1.5) in $[0, T] \times \mathbb{R}^{5}$, such that

$$
\sup _{0 \leq t \leq T}\left\|\left(u, u_{t}\right)\right\|_{\dot{H}^{1 / 2} \times \dot{H}^{-1 / 2}\left(\mathbb{R}^{5}\right)}+\|u\|_{L_{t, x}^{3}\left([0, T] \times \mathbb{R}^{5}\right)} \leq C(A) .
$$

In addition, if $A>0$ is small enough, we can take $T=\infty$.

We define $T_{+}\left(u_{0}, u_{1}\right):=\sup I$, where $I$ is the maximal interval of existence of the solution $u$.

Lemma 2.8 (Standard blowup criterion). Suppose $u$ is the solution to the Cauchy problem (1.5) with initial data $\left(u_{0}, u_{1}\right) \in \dot{H}^{1 / 2} \times \dot{H}^{-1 / 2}\left(\mathbb{R}^{5}\right)$ and $T_{+}\left(u_{0}, u_{1}\right)<\infty$. Then we have

$$
\|u\|_{L_{t, x}^{3}\left(\left[0, T_{+}\left(u_{0}, u_{1}\right)\right) \times \mathbb{R}^{5}\right)}=\infty .
$$

The proof is standard and similar to the energy critical case in [13.

We end this section by recalling the stability lemma for the Cauchy problem (1.5), which plays an important role in the Section 5 .

Theorem 3 (Perturbation theory [3]). Let $I \subset \mathbb{R}$ be a time interval with $0 \in I$. Let $\left(u_{0}, u_{1}\right) \in$ $\dot{H}^{1 / 2} \times \dot{H}^{-1 / 2}\left(\mathbb{R}^{5}\right)$ and some constants $M, A, A^{\prime}>0$ be given. Let $\tilde{u}$ be defined on $I \times \mathbb{R}^{5}$ and satisfy

$$
\sup _{t \in I}\left\|\left(\tilde{u}, \partial_{t} \tilde{u}\right)\right\|_{\dot{H}^{1 / 2} \times \dot{H}^{-1 / 2}\left(\mathbb{R}^{5}\right)} \leq A
$$

and

$$
\|\tilde{u}\|_{L_{t, x}^{3}\left(I \times \mathbb{R}^{5}\right)} \leq M
$$

Assume that $\partial_{t t} \tilde{u}-\Delta \tilde{u}=-|\tilde{u}| \tilde{u}+e$ on $I \times \mathbb{R}^{5}$,

$$
\left\|\left(u_{0}-\tilde{u}(0), u_{1}-\partial_{t} \tilde{u}(0)\right)\right\|_{\dot{H}^{1 / 2} \times \dot{H}^{-1 / 2}\left(\mathbb{R}^{5}\right)} \leq A^{\prime}
$$

and that

$$
\|e\|_{L_{t, x}^{3 / 2}\left(I \times \mathbb{R}^{5}\right)}+\left\|S(t)\left[\left(\tilde{u}(0), \partial_{t} \tilde{u}(0)\right)-\left(u_{0}, u_{1}\right)\right]\right\|_{L_{t, x}^{3}\left(I \times \mathbb{R}^{5}\right)}<\varepsilon .
$$

Then, there exist $\beta>0$ and $\varepsilon_{0}=\varepsilon_{0}\left(M, A, A^{\prime}\right)>0$ such that, for $0<\varepsilon<\varepsilon_{0}$, there exists a solution $u$ to (1.5) in I such that $\left(u(0), \partial_{t} u(0)\right)=\left(u_{0}, u_{1}\right)$, with

$$
\|u\|_{L_{t, x}^{3}\left(I \times \mathbb{R}^{5}\right)} \leq C\left(M, A, A^{\prime}\right),
$$

and

$$
\sup _{t \in I}\left\|\left(\partial_{t} \tilde{u}(t), \tilde{u}(t)\right)-\left(u, \partial_{t} u(t)\right)\right\|_{\dot{H}^{1 / 2} \times \dot{H}^{-1 / 2}\left(\mathbb{R}^{5}\right)} \leq C\left(M, A, A^{\prime}\right)\left(A^{\prime}+\varepsilon^{\beta}\right) .
$$




\section{Decomposition of the Solution And Global Well-Posedness}

In this section, we will prove that for any given initial data $\left(u_{0}, u_{1}\right) \in \dot{B}_{1,1}^{3} \times \dot{B}_{1,1}^{2}\left(\mathbb{R}^{5}\right)$, then the corresponding solution $u$ to the equation (1.5) is globally well-posed. To prove this, we first show the solution $u$ belongs to some suitable Strichartz-type spaces on a local time interval. Then, we split it into two parts: $u=v+w$. Based on the inhomogeneous Strichartz estimates (2.28), we will derive a decay property for $v$ and prove that $w$ is in the energy space $\dot{H}^{1} \times L^{2}\left(\mathbb{R}^{5}\right)$. We remark that the constants in " $\lesssim$ " in this section depend upon $\left\|\left(u_{0}, u_{1}\right)\right\|_{\dot{B}_{1,1}^{3} \times \dot{B}_{1,1}^{2}\left(\mathbb{R}^{5}\right)}$.

For the sake of simplicity, we denote $F(u)=|u| u$. Recall that $u_{\lambda}$ is also a solution to the Cauchy problem (1.5) with initial data $\left(\lambda^{2} u_{0}(\lambda x), \lambda^{3} u_{1}(\lambda x)\right)$, where

$$
u_{\lambda}(t, x)=\lambda^{2} u(\lambda t, \lambda x) .
$$

Given $\left(u_{0}, u_{1}\right) \in \dot{B}_{1,1}^{3} \times \dot{B}_{1,1}^{2}\left(\mathbb{R}^{5}\right)$, for any $\eta>0$, there exists $j_{0}=j_{0}\left(u_{0}, u_{1}, \eta\right)<\infty$ such that

$$
\sum_{j \geq j_{0}} 2^{2 j}\left\|P_{j} u_{0}\right\|_{L^{1}\left(\mathbb{R}^{5}\right)}<\eta
$$

Replace $u$ by $u_{\lambda}$ for $\lambda=2^{-j_{0}}$, then we have

$$
\sum_{j \geq 0} 2^{3 j}\left\|P_{j} u_{0}\right\|_{L^{1}\left(\mathbb{R}^{5}\right)}+\sum_{j \geq 0} 2^{2 j}\left\|P_{j} u_{1}\right\|_{L^{1}\left(\mathbb{R}^{5}\right)}<\eta .
$$

Lemma 3.1. Let $\epsilon_{0}>0$ be a small constant and $\eta \ll \epsilon_{0}$. If the initial data $\left(u_{0}, u_{1}\right) \in \dot{B}_{1,1}^{3} \times \dot{B}_{1,1}^{2}\left(\mathbb{R}^{5}\right)$ satisfies (3.3) and $u$ is the solution to (1.5) with initial data $\left(u_{0}, u_{1}\right)$ given by Theorem 2 , then there exists

such that

$$
\delta=\delta\left(\epsilon_{0},\left\|\left(u_{0}, u_{1}\right)\right\|_{\dot{B}_{1,1}^{3} \times \dot{B}_{1,1}^{2}}\right)>0
$$

$$
\begin{aligned}
\|u\|_{L_{t, x}^{3}\left([-\delta, \delta] \times \mathbb{R}^{5}\right)} & \leq \sum_{j \in \mathbb{Z}}\left\|P_{j} u\right\|_{L_{t, x}^{3}\left([-\delta, \delta] \times \mathbb{R}^{5}\right)} \lesssim \epsilon_{0}, \\
\|u\|_{L_{t}^{\infty}\left([-\delta, \delta] ; \dot{B}_{2,1}^{1 / 2}\left(\mathbb{R}^{5}\right)\right)} & \lesssim \sum_{j \in \mathbb{Z}}\left\|P_{j} u\right\|_{L_{t}^{\infty} \dot{H}^{1 / 2}\left([-\delta, \delta] \times \mathbb{R}^{5}\right)} \lesssim 1 .
\end{aligned}
$$

Proof. By Strichartz's estimates in Proposition 2.6 and (3.3), we obtain

$$
\left\|S(t) P_{\geq 0}\left(u_{0}, u_{1}\right)\right\|_{L_{t, x}^{3}\left(\mathbb{R} \times \mathbb{R}^{5}\right)} \leq \frac{1}{2} \epsilon_{0}
$$

On the other hand, for every $t \in \mathbb{R}$, by Bernstein, we have

$$
\left\|S(t) P_{\leq 0}\left(u_{0}, u_{1}\right)\right\|_{L_{x}^{3}\left(\mathbb{R}^{5}\right)} \lesssim 1
$$

Hence, taking $\delta$ small enough, we have,

$$
\left\|S(t)\left(u_{0}, u_{1}\right)\right\|_{L_{t, x}^{3}\left([-\delta, \delta] \times \mathbb{R}^{5}\right)} \leq \epsilon_{0}
$$

Then, by the Strichartz estimates, we have

$$
\|u\|_{L_{t, x}^{3}\left([-\delta, \delta] \times \mathbb{R}^{5}\right)} \lesssim\left\|S(t)\left(u_{0}, u_{1}\right)\right\|_{L_{t, x}^{3}\left([-\delta, \delta] \times \mathbb{R}^{5}\right)}+\|u\|_{L_{t, x}^{3}\left([-\delta, \delta] \times \mathbb{R}^{5}\right)}^{2},
$$

from which, by a standard continuity argument, we deduce that

$$
\|u\|_{L_{t, x}^{3}\left([-\delta, \delta] \times \mathbb{R}^{5}\right)} \lesssim \epsilon_{0}
$$

Let

$$
a_{k}=\left\|P_{k} u\right\|_{L_{t, x}^{3}\left([-\delta, \delta] \times \mathbb{R}^{5}\right)}+2^{\frac{1}{2} k}\left\|P_{k} u\right\|_{L_{t}^{\infty} L_{x}^{2}\left([-\delta, \delta] \times \mathbb{R}^{5}\right)}+2^{\frac{1}{4} k}\left\|P_{k} u\right\|_{L_{t}^{6} L_{x}^{\frac{12}{5}}\left([-\delta, \delta] \times \mathbb{R}^{5}\right)},
$$




$$
b_{k}=2^{\frac{k}{2}}\left\|P_{k} u_{0}\right\|_{L_{x}^{2}}+2^{-\frac{k}{2}}\left\|P_{k} u_{1}\right\|_{L_{x}^{2}} .
$$

By the Young inequality, it suffices to show there is a recurrence relation

$$
a_{k} \lesssim b_{k}+\epsilon_{0} \sum_{j} 2^{-\frac{1}{4}|j-k|} a_{j} .
$$

To prove this, making use of the Strichartz estimates, we have

$$
a_{k} \lesssim b_{k}+2^{\frac{k}{4}}\left\|P_{k} F(u)\right\|_{L_{t}^{2} L_{x}^{\frac{4}{3}}\left([0, \delta] \times \mathbb{R}^{5}\right)} .
$$

First, we consider the low frequency part of the second term in the right hand of (3.14). By Lemma 2.4 and Hölder, we have

$$
\begin{aligned}
& \left\|P_{k} F\left(u_{\leq k}\right)\right\|_{L_{t}^{2} L_{x}^{\frac{4}{3}}\left([-\delta, \delta] \times \mathbb{R}^{5}\right)} \\
\lesssim & 2^{-\frac{1}{2} k}\left\|P_{k}\left|\nabla_{x}\right|^{\frac{1}{2}} F\left(u_{\leq k}\right)\right\|_{L_{t}^{2} L_{x}^{\frac{4}{3}}\left([-\delta, \delta] \times \mathbb{R}^{5}\right)} \\
\lesssim & 2^{-\frac{1}{2} k}\|u\|_{L_{t, x}^{3}\left([-\delta, \delta] \times \mathbb{R}^{5}\right)}\left\||\nabla|^{\frac{1}{2}}\left(P_{\leq k} u\right)\right\|_{L_{t}^{6} L_{x}^{\frac{12}{5}}\left([-\delta, \delta] \times \mathbb{R}^{5}\right)} \\
\lesssim & \|u\|_{L_{t, x}^{3}\left([-\delta, \delta] \times \mathbb{R}^{5}\right)} \sum_{j \leq k} 2^{-\frac{k-j}{2}}\left\|P_{j} u\right\|_{L_{t}^{6} L_{x}^{\frac{12}{5}}\left([-\delta, \delta] \times \mathbb{R}^{5}\right)},
\end{aligned}
$$

from which it follows that

$$
\begin{aligned}
& 2^{\frac{k}{4}}\left\|P_{k} F\left(u_{\leq k}\right)\right\|_{L_{t}^{2} L_{x}^{\frac{4}{3}}\left([-\delta, \delta] \times \mathbb{R}^{5}\right)} \\
\lesssim & \epsilon_{0} \sum_{j \leq k} 2^{-\frac{k-j}{4}} 2^{\frac{1}{4} j}\left\|P_{j} u\right\|_{L_{t}^{6} L_{x}^{\frac{12}{5}}\left([-\delta, \delta] \times \mathbb{R}^{5}\right)} \lesssim \epsilon_{0} \sum_{j \leq k} 2^{-\frac{k-j}{4}} a_{j} .
\end{aligned}
$$

On the other hand, by Hölder's inequality,

$$
\begin{aligned}
& 2^{\frac{k}{4}}\left\|P_{k}\left(F(u)-F\left(u_{\leq k}\right)\right)\right\|_{L_{t}^{2} L_{x}^{\frac{4}{3}}\left([-\delta, \delta] \times \mathbb{R}^{5}\right)} \\
\lesssim & \|u\|_{L_{t, x}^{3}\left([-\delta, \delta] \times \mathbb{R}^{5}\right)} 2^{\frac{1}{4} k}\left\|P_{\geq k+1} u\right\|_{L_{t}^{6} L_{x}^{\frac{12}{5}}\left([-\delta, \delta] \times \mathbb{R}^{5}\right)} \\
\lesssim & \epsilon_{0} \sum_{j \geq k+1} 2^{-\frac{j-k}{4}} 2^{\frac{1}{4} j}\left\|P_{j} u\right\|_{L_{t}^{6} L_{x}^{\frac{12}{5}}\left([-\delta, \delta] \times \mathbb{R}^{5}\right)} \lesssim \epsilon_{0} \sum_{j \geq k+1} 2^{-\frac{j-k}{4}} a_{j} .
\end{aligned}
$$

Then the recurrence relation (3.13) follows from (3.16) and (3.17).

Remark that by the inequality (3.5), the inequalities (3.16) and (3.17) yield that

$$
\sum_{k \in \mathbb{Z}} 2^{\frac{k}{4}}\left\|P_{k}(F(u))\right\|_{L_{t}^{2} L_{x}^{\frac{4}{3}}\left([0, \delta] \times \mathbb{R}^{5}\right)} \lesssim \epsilon_{0}
$$

As an application of Lemma 3.1 and the radial Sobolev inequality (2.2), we will see that the solution $u$ posses some space decay property in the region $\{|x| \geq|t|+C\}$ for some large constant $C>0$. Let $\chi(x)$ be a smooth cutoff function such that $\chi(x)=1$ for $|x| \leq \frac{1}{2}$ and $\chi(x)=0$ for $|x| \geq 1$. By Lemma 2.3 , there exists a dyadic integer $R=R\left(u_{0}, u_{1}, \epsilon_{0}\right)$ such that $\left\|\left(1-\chi\left(\frac{x}{R}\right)\right)\left(u_{0}, u_{1}\right)\right\|_{\dot{B}_{2,1}^{1 / 2} \times \dot{B}_{2,1}^{-1 / 2}\left(\mathbb{R}^{5}\right)} \leq \epsilon_{0}$. Then by scaling, we have

$$
\left\|(1-\chi(2 x))\left((2 R)^{2} u_{0}(2 R x),(2 R)^{3} u_{1}(2 R x)\right)\right\|_{\dot{B}_{2,1}^{1 / 2} \times \dot{B}_{2,1}^{-1 / 2}\left(\mathbb{R}^{5}\right)} \leq \epsilon_{0} .
$$

By abuse of notations, we will still use $\left(u_{0}, u_{1}\right)$ to represent the initial data $\left((2 R)^{2} u_{0}(2 R x),(2 R)^{3} u_{1}(2 R x)\right)$. Then we have,

$$
\left\|(1-\chi(2 x))\left(u_{0}, u_{1}\right)\right\|_{\dot{B}_{2,1}^{1 / 2} \times \dot{B}_{2,1}^{-1 / 2}\left(\mathbb{R}^{5}\right)} \leq \epsilon_{0}
$$


In addition, by Lemma 3.1, we have

$$
\begin{aligned}
\|u\|_{L_{t, x}^{3}\left(\left[-\frac{\delta}{2 R}, \frac{\delta}{2 R}\right] \times \mathbb{R}^{5}\right)} & \lesssim \epsilon_{0}, \\
\|u\|_{L_{t}^{\infty}\left(\left[-\frac{\delta}{2 R}, \frac{\delta}{2 R}\right] ; \dot{B}_{2,1}^{\frac{1}{2}}\left(\mathbb{R}^{5}\right)\right)} & \lesssim 1 .
\end{aligned}
$$

Lemma 3.2. Let $J \subset \mathbb{R}$ be an interval such that $u$ is a solution to (1.5) on $J$. Then we have

$$
\|u\|_{L_{t, x}^{3}\left(\left\{(t, x) \in J \times \mathbb{R}^{5}:|x| \geq|t|+\frac{1}{2}\right\}\right)}+\sup _{t \in J}\left\||x|^{2} u(t, x)\right\|_{L_{x}^{\infty}\left(\left\{x \in \mathbb{R}^{5}:|x| \geq|t|+\frac{1}{2}\right\}\right)} \lesssim \epsilon_{0} .
$$

Proof. Let $U(t, x)$ be the solution to (1.5) with initial data $(1-\chi(2 x))\left(u_{0}(x), u_{1}(x)\right)$. Employing Theorem 2 and arguing by similar arguments in Lemma 3.1, one can deduce (3.14) when $u$ is replaced by $U$ and $[-\delta, \delta]$ is replaced by $\mathbb{R}$. Thus, we have

$$
\|U\|_{L_{t, x}^{3}\left(\mathbb{R} \times \mathbb{R}^{5}\right)}+\|U\|_{L_{t}^{\infty} \dot{B}_{2,1}^{\frac{1}{2}}\left(\mathbb{R} \times \mathbb{R}^{5}\right)} \lesssim \epsilon_{0}
$$

Due to the finite propagation speed property of the wave equation (1.5), we have $u(t, x)=U(t, x)$ when $|x| \geq|t|+\frac{1}{2}$. Then (3.23) follows from (3.24) and the radial Sobolev inequality (2.2).

Next, we want to show the following local properties, which will play an important role in the following Subsection 3.2. Unlike the case of the three dimension in [6], we will make use of the inhomogeneous Strichartz estimates (2.28) to conquer the difficulties caused by the higher order decay of time.

Lemma 3.3. If $\epsilon_{0}$ is sufficiently small and $\delta$ is given in Lemma 3.1, then, for $3<r<4$, we have

$$
\sup _{-\frac{2 \delta}{2 R}<t<\frac{2 \delta}{2 R}} t^{\frac{2 r-5}{r}}\|u\|_{L_{x}^{r}\left(\mathbb{R}^{5}\right)}+\|u\|_{L_{t}^{\frac{5}{4}} L_{x}^{\frac{25}{6}}\left(\left[0, \frac{\delta}{2 R}\right] \times \mathbb{R}^{5}\right)} \lesssim 1
$$

We remark that for $3<r<\infty$, the space $L_{t}^{\frac{r}{2 r-5}} L_{x}^{r}\left(\mathbb{R} \times \mathbb{R}^{5}\right)$ is $\dot{H}^{\frac{1}{2}}$-critical but not admissible.

Proof. First, we consider the estimates for the linear part. Utilizing dispersive estimate (2.20), we have

$$
\left\|S(t) P_{j}\left(u_{0}, u_{1}\right)\right\|_{L_{x}^{\infty}\left(\mathbb{R}^{5}\right)} \lesssim \frac{1}{t^{2}}\left[2^{3 j}\left\|P_{j} u_{0}\right\|_{L^{1}\left(\mathbb{R}^{5}\right)}+2^{2 j}\left\|P_{j} u_{1}\right\|_{L^{1}\left(\mathbb{R}^{5}\right)}\right]
$$

By Bernstein, we have

$$
\left\|S(t) P_{j}\left(u_{0}, u_{1}\right)\right\|_{L_{x}^{2}\left(\mathbb{R}^{5}\right)} \lesssim\left\|P_{j} u_{0}\right\|_{L_{x}^{2}\left(\mathbb{R}^{5}\right)}+2^{-j}\left\|P_{j} u_{1}\right\|_{L_{x}^{2}\left(\mathbb{R}^{5}\right)} \lesssim 2^{\frac{5}{2} j}\left\|P_{j} u_{0}\right\|_{L^{1}\left(\mathbb{R}^{5}\right)}+2^{\frac{3}{2} j}\left\|P_{j} u_{1}\right\|_{L^{1}\left(\mathbb{R}^{5}\right)} .
$$

Interpolating this inequality with the estimate (3.26) yields that,

$$
\left\|S(t) P_{j}\left(u_{0}, u_{1}\right)\right\|_{L_{x}^{r}\left(\mathbb{R}^{5}\right)} \lesssim t^{-2\left(1-\frac{2}{r}\right)} 2^{-\frac{j}{r}}\left[2^{3 j}\left\|P_{j} u_{0}\right\|_{L^{1}\left(\mathbb{R}^{5}\right)}+2^{2 j}\left\|P_{j} u_{1}\right\|_{L^{1}\left(\mathbb{R}^{5}\right)}\right] .
$$

On the other hand, for $r \geq \frac{5}{2}$, employing Bernstein's estimates, we have

$$
\begin{aligned}
\left\|S(t) P_{j}\left(u_{0}, u_{1}\right)\right\|_{L_{x}^{r}\left(\mathbb{R}^{5}\right)} & \lesssim 2^{5\left(\frac{1}{2}-\frac{1}{r}\right) j}\left\|S(t) P_{j}\left(u_{0}, u_{1}\right)\right\|_{L_{x}^{2}\left(\mathbb{R}^{5}\right)} \\
& \lesssim 2^{\left(2-\frac{5}{r}\right) j}\left[2^{3 j}\left\|P_{j} u_{0}\right\|_{L^{1}\left(\mathbb{R}^{5}\right)}+2^{2 j}\left\|P_{j} u_{1}\right\|_{L^{1}\left(\mathbb{R}^{5}\right)}\right] .
\end{aligned}
$$

This estimate and (3.28) yield that, for $r \geq \frac{5}{2}$,

$$
\sup _{t \in \mathbb{R}} t^{\frac{2 r-5}{r}}\left\|S(t)\left(u_{0}, u_{1}\right)\right\|_{L_{x}^{r}\left(\mathbb{R}^{5}\right)}+\left\|S(t)\left(u_{0}, u_{1}\right)\right\|_{L_{t}^{\frac{r}{2 r-5}} L_{x}^{r}\left(\mathbb{R} \times \mathbb{R}^{5}\right)} \lesssim\left\|\left(u_{0}, u_{1}\right)\right\|_{\dot{B}_{1,1}^{3} \times \dot{B}_{1,1}^{2}\left(\mathbb{R}^{5}\right)} .
$$


By the reversal property of the wave equation, it suffices to prove (3.25) for $t \geq 0$. Using the inhomogeneous Strichartz estimates (2.28), Lemma 2.4, Lemma 3.1, and Hölder, we have

$$
\begin{aligned}
& \left\|\int_{0}^{t} S(t-\tau)(0, F(u(\tau))) d \tau\right\|_{L_{t}^{\frac{5}{4}} L_{x}^{\frac{25}{6}}\left(\left[0, \frac{\delta}{2 R}\right] \times \mathbb{R}^{5}\right)} \\
& \lesssim\left\||\nabla|^{\frac{1}{4}} F(u)\right\|_{L_{t}^{\frac{30}{29}} L_{x}^{\frac{300}{197}}\left(\left[0, \frac{\delta}{2 R}\right] \times \mathbb{R}^{5}\right)}\|\|\left\|_{L_{t, x}^{3}\left(\left[0, \frac{\delta}{2 R}\right] \times \mathbb{R}^{5}\right)}^{1 / 2}\right\| u \|_{L_{t}^{\frac{5}{4}} L_{x}^{\frac{25}{6}}\left(\left[0, \frac{\delta}{2 R}\right] \times \mathbb{R}^{5}\right)} . \\
& \lesssim\left\||\nabla| \frac{1}{2} u\right\|_{L_{t}^{\infty} L_{x}^{2}\left(\left[0, \frac{\delta}{2 R}\right] \times \mathbb{R}^{5}\right)}^{1 / 2}
\end{aligned}
$$

This estimates together with the estimate (3.30) yields

$$
\|u\|_{L_{t}^{\frac{5}{4}} L_{x}^{\frac{25}{6}}\left(\left[0, \frac{\delta}{2 R}\right] \times \mathbb{R}^{5}\right)} \lesssim 1
$$

provided $0<\epsilon_{0} \ll \min \left(1,\left\|\left(u_{0}, u_{1}\right)\right\|_{\dot{B}_{1,1}^{3} \times \dot{B}_{1,1}^{2}\left(\mathbb{R}^{5}\right)}^{-1}\right)$.

Let $c \in(0,1)$ to be chosen later. First, employing the dispersive estimate (2.20), Lemma 2.4, and interpolation, for $r \in(3,4)$, we have

$$
\begin{aligned}
& \sup _{t \in\left[0, \frac{\delta}{2 R}\right]} t^{\frac{2 r-5}{r}}\left\|\int_{(1-c) t}^{t} S(t-\tau)(0, F(u(\tau))) d \tau\right\|_{L^{r}\left(\mathbb{R}^{5}\right)} \\
& \lesssim \sup _{t \in\left[0, \frac{\delta}{2 R}\right]} t^{\frac{2 r-5}{r}} \int_{(1-c) t}^{t} \frac{1}{(t-\tau)^{2-\frac{4}{r}}}\left\||\nabla|^{2-\frac{6}{r}} F(u(\tau))\right\|_{L^{r^{\prime}\left(\mathbb{R}^{5}\right)}} d \tau \\
& \lesssim \sup _{t \in\left[0, \frac{\delta}{2 R}\right]} t^{\frac{2 r-5}{r}} \int_{(1-c) t}^{t} \frac{1}{(t-\tau)^{2-\frac{4}{r}}}\|u(\tau)\|_{L^{r}\left(\mathbb{R}^{5}\right)}^{\frac{r-1}{2 r-5}}\|u(\tau)\|_{\dot{H}_{x}^{\frac{1}{2}}\left(\mathbb{R}^{5}\right)}^{4-\frac{12}{r}}\|u(\tau)\|_{L_{x}^{\frac{5}{2}}\left(\mathbb{R}^{5}\right)}^{\left(\frac{12}{2 r-5}\right)} d \tau \\
& \lesssim\left.\sup _{t \in\left[0, \frac{\delta}{2 R}\right]} t^{\frac{2 r-5}{r}}\|u(t)\|_{L^{r}\left(\mathbb{R}^{5}\right)}\right)^{\frac{r-1}{2 r-5}}\|u\|_{L_{t}^{\infty} \dot{H}_{x}^{\frac{1}{2}}\left(\left[0, \frac{\delta}{2 R}\right] \times \mathbb{R}^{5}\right)}^{4-\frac{12}{r}}\|u\|_{L_{t}^{\infty} L_{x}^{\frac{5}{2}}\left(\left[0, \frac{\delta}{2 R}\right] \times \mathbb{R}^{5}\right)}^{\left(\frac{12}{2 r-5}\right)} \cdot \int_{(1-c) t}^{t} \frac{1}{(t-\tau)^{2-\frac{4}{r}}} t^{1-\frac{4}{r}} d \tau \\
& \lesssim c^{4 / r-1}\left(\sup _{t \in\left[0, \frac{\delta}{2 R}\right]} t^{\frac{2 r-5}{r}}\|u(t)\|_{L^{r}\left(\mathbb{R}^{5}\right)}\right)^{\frac{r-1}{2 r-5}} .
\end{aligned}
$$

For the remainder part, we utilize the dispersive estimate (2.20), Lemma 2.4, the Hölder inequality, and interpolation to obtain

$$
\begin{aligned}
& t^{\frac{2 r-5}{r}}\left\|\int_{0}^{(1-c) t} S(t-\tau)(0, F(u(\tau))) d \tau\right\|_{L^{r}\left(\mathbb{R}^{5}\right)} \\
\lesssim & t^{\frac{2 r-5}{r}} \int_{0}^{(1-c) t} \frac{1}{(t-\tau)^{2-\frac{4}{r}}}\left\||\nabla|^{2-\frac{6}{r}} F(u(\tau))\right\|_{L_{x}^{r^{\prime}\left(\mathbb{R}^{5}\right)}} d \tau \\
\lesssim & \left.c^{\frac{4}{r}-2}\|\| \nabla\right|^{2-\frac{6}{r}} F(u)\left\|_{L_{t, x}^{r^{\prime}}\left(\mathbb{R} \times \mathbb{R}^{5}\right)}\right\| \|^{4-\frac{12}{r}} \\
\lesssim & c^{\frac{4}{r}-2}\|u\|_{L_{t}^{\infty} \dot{H}_{x}^{\frac{1}{2}}\left(\mathbb{R} \times \mathbb{R}^{5}\right)}\|u\|_{L_{t, x}^{3}\left(\mathbb{R} \times \mathbb{R}^{5}\right)}^{\frac{12}{2 r-5}} L_{x}^{r}\left(\mathbb{R} \times \mathbb{R}^{5}\right) \\
\lesssim & c^{\frac{4}{r}-2} \epsilon_{0}^{\frac{159}{7 r}-\frac{39}{7}},
\end{aligned}
$$


where in the last step we used the fact that

$$
\|u\|_{L_{t}^{\frac{r}{2 r-5}} L_{x}^{r}\left(\left[0, \frac{\delta}{2 R}\right] \times \mathbb{R}^{5}\right)} \lesssim\|u\|_{L_{t, x}^{3}\left(\left[0, \frac{\delta 5}{2 R}\right] \times \mathbb{R}^{5}\right)}^{\left.\frac{1}{7}-\frac{6}{25}\right)}\|u\|_{L_{t}^{\frac{25}{4}} L_{x}^{\frac{25}{6}}\left(\left[1-\frac{3}{r}\right)\right.} \lesssim \epsilon_{0}^{\frac{75}{7}\left(\frac{1}{r}-\frac{6}{25}\right)}
$$

Hence, by (3.30) and (3.32)-(3.34), taking $c>0$ small enough and using a continuity method, there exists $\epsilon_{0}=\epsilon_{0}\left(\left\|\left(u_{0}, u_{1}\right)\right\|_{\dot{B}_{1,1}^{3} \times \dot{B}_{1,1}^{2}}\right)>0$ such that

$$
\sup _{t \in\left[0, \frac{\delta}{2 R}\right]} t^{\frac{2 r-5}{r}}\|u\|_{L_{x}^{r}} \lesssim 1, \text { for } 3<r<4 .
$$

We denote $\delta_{1}=\frac{\delta}{2 R}$ for simplicity. Let $\psi \in C_{0}^{\infty}\left(\mathbb{R}^{5}\right)$ supported in $|x| \leq \frac{\delta_{1}}{10}$ and $\psi(x)=1$ when $|x| \leq \frac{\delta_{1}}{20}$. Then we can assume that $|(\nabla \psi)(x)| \lesssim \frac{1}{\delta_{1}}$. For $t \geq \delta_{1}$, we split $u(t, x)=v(t, x)+w(t, x)$, where

$$
v(t)=S(t)\left(\psi u_{0}, \psi u_{1}\right)-\int_{0}^{\delta_{1} / 10} S(t-\tau)(0, \psi F(u(\tau))) d \tau .
$$

We will prove that $v$ has a decay property and $w$ is with finite energy.

\subsection{The decay part of the solution $u$.}

Lemma 3.4. For $t \geq \delta_{1}$, we have

$$
\|v(t)\|_{L_{x}^{\infty}\left(\mathbb{R}^{5}\right)} \lesssim \delta_{1}^{-\frac{1}{2}} t^{-2}
$$

In addition, we have

$$
\|v\|_{L_{t}^{\infty}\left(\mathbb{R} ; \dot{B}_{2,1}^{\frac{1}{2}}\left(\mathbb{R}^{5}\right)\right)}+\|v\|_{L_{t, x}^{3}\left(\mathbb{R} \times \mathbb{R}^{5}\right)} \lesssim \delta_{1}^{-\frac{1}{2}}
$$

Proof. We first estimate the linear part of $v$. By the Huygens principle, the radial Sobolev inequality (2.2) and Lemma 2.3. we have, for $t \geq \delta_{1}$

$$
\left\|S(t)\left(\psi u_{0}, \psi u_{1}\right)\right\|_{L_{x}^{\infty}\left(\mathbb{R}^{5}\right)} \lesssim \frac{1}{t^{2}}\left\|\left(u_{0}, u_{1}\right)\right\|_{\dot{B}_{2,1}^{\frac{1}{2}} \times \dot{B}_{2,1}^{-\frac{1}{2}}} \lesssim \frac{1}{t^{2}}
$$

For the second part of $v$ and $t \geq \delta_{1}$, using the Radial Sobolev inequality (2.2), the Huygens principle and the Strichartz estimates, we obtain

$$
\begin{aligned}
& \left\|\int_{0}^{\delta_{1} / 10} S(t-\tau)(0, \psi F(u(\tau))) d \tau\right\|_{L_{x}^{\infty}\left(\mathbb{R}^{5}\right)} \\
\lesssim & \frac{1}{t^{2}}\left\|\int_{0}^{\delta_{1} / 10} S(t-\tau)(0, \tilde{\chi} F(u(\tau))) d \tau\right\|_{\dot{B}_{2,1}^{\frac{1}{2}}\left(\mathbb{R}^{5}\right)} \\
\lesssim & \frac{1}{t^{2}}\left\|\int_{0}^{\delta_{1} / 10} \frac{\sin (\tau|\nabla|)}{|\nabla|}(\psi F(u(\tau))) d \tau\right\|_{\dot{B}_{2,1}^{\frac{1}{2}}}+\frac{1}{t^{2}}\left\|\int_{0}^{\delta_{1} / 10} \frac{\cos (\tau|\nabla|)}{|\nabla|}(\psi F(u(\tau))) d \tau\right\|_{\dot{B}_{2,1}^{\frac{1}{2}}} \\
\lesssim & \frac{1}{t^{2}} \sum_{j<0} 2^{-\frac{1}{2} j}\left\|P_{j}[\psi F(u(\tau))]\right\|_{L_{t}^{1} L_{x}^{2}\left(\left[0, \delta_{1} / 10\right] \times \mathbb{R}^{5}\right)} \\
& +\frac{1}{t^{2}} \sum_{j \geq 0} 2^{\frac{1}{4} j}\left\|P_{j}[\psi F(u(\tau))]\right\| \|_{L_{t}^{2} L_{x}^{\frac{4}{3}}\left(\left[0, \delta_{1} / 10\right] \times \mathbb{R}^{5}\right)}
\end{aligned}
$$

For the low frequency part (3.41), by Bernstein's estimates and Hölder's inequality, we have 


$$
t^{2}(\underline{3.41)}) \lesssim \sum_{j<0} 2^{\frac{j}{3}}\|F(u)\|_{L_{t}^{1} L_{x}^{\frac{3}{2}}\left(\left[0, \delta_{1} / 10\right] \times \mathbb{R}^{5}\right)} \lesssim \delta_{1}^{\frac{1}{3}}\|u\|_{L_{t, x}^{3}\left(\left[0, \delta_{1} / 10\right] \times \mathbb{R}^{5}\right)}^{2} \lesssim 1 .
$$

For (3.42), it suffices to estimate

$$
\begin{aligned}
& \sum_{j \geq 0} 2^{\frac{1}{4} j}\left\|\left[P_{j}, \psi\right] F(u(\tau))\right\|_{L_{t}^{2} L_{x}^{\frac{4}{3}}\left(\left[0, \delta_{1} / 10\right] \times \mathbb{R}^{5}\right)} \\
+ & \sum_{j \geq 0} 2^{\frac{1}{4} j}\left\|\psi P_{j} F(u(\tau))\right\|_{L_{t}^{2} L_{x}^{\frac{4}{3}}\left(\left[0, \delta_{1} / 10\right] \times \mathbb{R}^{5}\right)} .
\end{aligned}
$$

For (3.44), by commutator estimates, the Young inequality, the Sobolev embedding and Lemma 3.1 , we have

$$
\begin{aligned}
(\underline{3.44}) & \lesssim \sum_{j \geq 0} 2^{-\frac{3}{4} j} \delta_{1}^{-1}\left\|Q_{j} F(u(\tau))\right\|_{L_{\tau}^{2} L_{x}^{\frac{4}{3}}\left(\left[0, \delta_{1} / 10\right] \times \mathbb{R}^{5}\right)} \\
& \lesssim \delta_{1}^{-1} \sum_{j \geq 0} 2^{-\frac{3}{4} j} 2^{\frac{1}{4} j}\|F(u(\tau))\|_{L_{\tau}^{2} L_{x}^{5 / 4}\left(\left[0, \delta_{1} / 10\right] \times \mathbb{R}^{5}\right)} \\
& \lesssim \delta_{1}^{-\frac{1}{2}}\|u\|_{L_{t}^{\infty} L_{x}^{\frac{5}{2}}\left(\left[0, \delta_{1} / 10\right] \times \mathbb{R}^{5}\right)}^{2} \lesssim \delta_{1}^{-\frac{1}{2}}
\end{aligned}
$$

where

$$
Q_{j} f(x)=2^{6 j} \int_{\mathbb{R}^{5}}|y| \phi\left(2^{j} y\right)|f|(x-y) d y
$$

and in the first inequality we used the mean value theorem. For (3.45), by the estimate (3.18), we have

$$
(3.45) \lesssim \sum_{j \geq 0} 2^{\frac{1}{4} j}\left\|P_{j} F(u)\right\|_{L_{\tau}^{2} L_{x}^{\frac{4}{3}}} \lesssim \epsilon_{0}
$$

Hence, by (3.39)-(3.47), we have $\|v(t)\|_{L_{x}^{\infty}\left(\mathbb{R}^{5}\right)} \lesssim \delta_{1}^{-\frac{1}{2}} t^{-2}$.

Now we consider (3.38). For simplicity, we write

$$
\|v\|_{S(\mathbb{R})}:=\|v\|_{L_{t, x}^{3}\left(\mathbb{R} \times \mathbb{R}^{5}\right)}+\|v\|_{L_{t}^{\infty}\left(\mathbb{R} ; \dot{B}_{2,1}^{\frac{1}{2}}\left(\mathbb{R}^{5}\right)\right)} .
$$

For the linear part, by the Strichartz estimates and Lemma 2.3. we have

$$
\left\|S(t)\left(\psi u_{0}, \psi u_{1}\right)\right\|_{S(\mathbb{R})} \lesssim\left\|\left(u_{0}, u_{1}\right)\right\|_{\dot{B}_{2,1}^{1 / 2} \times \dot{B}_{2,1}^{-1 / 2}\left(\mathbb{R}^{5}\right)} \lesssim 1
$$

By the Strichartz estimates and repeating the arguments that deal with (3.40), we have

$$
\begin{aligned}
& \left\|\int_{0}^{\delta_{1} / 10} S(t-\tau)(0, \psi F(u(\tau))) d \tau\right\|_{S(\mathbb{R})} \\
\lesssim & \left\|\int_{0}^{\delta_{1} / 10} \frac{\sin (\tau|\nabla|)}{|\nabla|}(\psi F(u(\tau))) d \tau\right\|_{\dot{B}_{2,1}^{\frac{1}{2}}\left(\mathbb{R}^{5}\right)}+\left\|\int_{0}^{\delta_{1} / 10} \frac{\cos (\tau|\nabla|)}{|\nabla|}(\psi F(u(\tau))) d \tau\right\|_{\dot{B}_{2,1}^{\frac{1}{2}}\left(\mathbb{R}^{5}\right)} \\
\lesssim & \delta_{1}^{-\frac{1}{2}} .
\end{aligned}
$$

This completes the proof. 


\subsection{The energy part of the solution $u$.}

Lemma 3.5. We have

$$
\left\|\vec{w}\left(\delta_{1}\right)\right\|_{\dot{H}^{1} \times L^{2}\left(\mathbb{R}^{5}\right)} \lesssim \delta_{1}^{-1 / 2} .
$$

Proof. By the definition of $w$, for $t \geq \delta_{1}$, we have

$$
\begin{aligned}
w(t)= & S(t)\left((1-\psi) u_{0},(1-\psi) u_{1}\right)-\int_{0}^{\frac{\delta_{1}}{10}} S(t-\tau)(0,(1-\psi) F(u(\tau))) d \tau \\
& -\int_{\frac{\delta_{1}}{10}}^{t} S(t-\tau)(0, F(u(\tau))) d \tau .
\end{aligned}
$$

First, we consider the contribution of the third term of (3.50). Taking $r=\frac{50}{13}$ in (3.25), by interpolation, we have

$$
\begin{aligned}
\|u\|_{L_{t}^{2} L_{x}^{4}\left(\left[\frac{\delta_{1}}{10}, \delta_{1}\right] \times \mathbb{R}^{5}\right)}^{2} & =\int_{\delta_{1} / 10}^{\delta_{1}}\|u\|_{L_{x}^{\frac{50}{3}}\left(\mathbb{R}^{5}\right)}\|u\|_{L_{x}^{\frac{25}{6}}\left(\mathbb{R}^{5}\right)} d t \\
& \lesssim \delta_{1}^{-\frac{1}{2}} \sup _{t \in\left[\frac{\delta_{1}}{10}, \delta_{1}\right]}\left[t \frac{7}{10}\|u(t)\|_{L_{x}^{\frac{50}{13}}\left(\mathbb{R}^{5}\right)}\right]\|u\|_{L_{t}^{\frac{5}{4}} L_{x}^{\frac{25}{6}}\left(\left[\frac{\delta_{1}}{10}, \delta_{1}\right] \times \mathbb{R}^{5}\right)} \lesssim \delta_{1}^{-\frac{1}{2}} .
\end{aligned}
$$

From this inequality and Strichartz, we have

$$
\begin{aligned}
& \left\|\int_{\delta_{1} / 10}^{\delta_{1}} S\left(\delta_{1}-\tau\right)(0, F(u(\tau))) d \tau\right\|_{\dot{H}_{x}^{1}\left(\mathbb{R}^{5}\right)}+\left\|\partial_{t}\left[\int_{\delta_{1} / 10}^{t} S(t-\tau)(0, F(u(\tau))) d \tau\right]_{t=\delta_{1}}\right\|_{L_{x}^{2}\left(\mathbb{R}^{5}\right)} \\
\lesssim & \|F(u)\|_{L_{\tau}^{1} L_{x}^{2}\left(\left[\frac{\delta_{1}}{10}, \delta_{1}\right] \times \mathbb{R}^{5}\right)} \lesssim\|u\|_{L_{\tau}^{2} L_{x}^{4}\left(\left[\frac{\delta_{1}}{10}, \delta_{1}\right] \times \mathbb{R}^{5}\right)}^{2} \lesssim \delta_{1}^{-\frac{1}{2}} .
\end{aligned}
$$

By Strichartz, radial Sobolev inequality (2.2) and Hölder, the second term of (3.50) can be estimated as

$$
\begin{aligned}
& \left\|\left.\int_{0}^{\delta_{1} / 10} \vec{S}(t-\tau)(0,(1-\psi) F(u(\tau))) d \tau\right|_{t=\delta_{1}}\right\|_{\dot{H}^{1} \times L^{2}\left(\mathbb{R}^{5}\right)} \\
\lesssim & \|(1-\psi) F(u(\tau))\|_{L_{\tau}^{1} L_{x}^{2}\left(\left[0, \frac{\delta_{1}}{10}\right] \times \mathbb{R}^{5}\right)} \\
\lesssim & \delta_{1}^{\frac{1}{2}}\|(1-\psi) u\|_{L_{t, x}^{\infty}\left(\left[0, \frac{\delta_{1}}{10}\right] \times \mathbb{R}^{5}\right)}^{\frac{1}{2}}\|u\|_{L_{t, x}^{3}\left(\left[0, \frac{\delta_{1}}{10}\right] \times \mathbb{R}^{5}\right)}^{\frac{3}{2}} \\
\lesssim & \delta_{1}^{-\frac{1}{2}}\|u\|_{L_{t}^{\infty} \dot{B}_{2,1}^{1 / 2}}^{\frac{1}{2}} \lesssim \delta_{1}^{-1 / 2} .
\end{aligned}
$$

Hence, it remains to estimate

$$
\left\|(1-\psi)\left(u_{0}, u_{1}\right)\right\|_{\dot{H}^{1} \times L^{2}\left(\mathbb{R}^{5}\right)} .
$$

For $u_{0}$, by radial Sobolev inequality (2.2) and polar coordinates,

$$
\left\|(\nabla \psi) u_{0}\right\|_{L^{2}\left(\mathbb{R}^{5}\right)}^{2} \lesssim \delta_{1}^{-1} \int_{\delta_{1} / 100}^{2 \delta_{1}} \int_{\mathbb{S}^{4}} u_{0}^{2}(r) d \sigma(\omega) r^{4} d r \lesssim\left\|u_{0}\right\|_{\dot{B}_{2,1}^{\frac{1}{2}}\left(\mathbb{R}^{5}\right)}^{2} \lesssim 1 .
$$

By the inequality (2.13), we have

$$
\left\|(1-\psi) \partial_{r} u_{0}\right\|_{L_{x}^{2}\left(\mathbb{R}^{5}\right)} \lesssim \delta_{1}^{-\frac{1}{2}} .
$$

For $u_{1}$, by the inequality (2.8) and polar coordinates, one can deduce that

$$
\left\|(1-\psi) u_{1}\right\|_{L_{x}^{2}\left(\mathbb{R}^{5}\right)}^{2} \lesssim \int_{\frac{\delta_{1}}{10}}^{\infty} \int_{\mathbb{S}^{4}}\left|u_{1}(r)\right|^{2} r^{4} d \sigma(\omega) d r \lesssim \int_{\frac{\delta_{1}}{10}}^{\infty} r^{-2} d r \lesssim \delta_{1}^{-1} .
$$


This inequality together with (3.55) and (3.56) implies that

$$
\left\|(1-\psi)\left(u_{0}, u_{1}\right)\right\|_{\dot{H}^{1} \times L^{2}\left(\mathbb{R}^{5}\right)} \lesssim \delta_{1}^{-\frac{1}{2}}
$$

This completes the proof.

3.3. Global well-posedness. In this subsection, we prove that the solution $u$ is globally wellposed. We emphasize that the constants in " $\lesssim$ " in this subsection depend only upon $\delta_{1}$ and $\left\|\left(u_{0}, u_{1}\right)\right\|_{\dot{B}_{1,1}^{3} \times \dot{B}_{1,1}^{2}\left(\mathbb{R}^{5}\right)}$.

Theorem 4. Let $u$ be the solution to (1.5) with initial data $\left(u_{0}, u_{1}\right) \in \dot{B}_{1,1}^{3} \times \dot{B}_{1,1}^{2}$. Then $u$ is globally well-posed and such that for any compact interval $J \subset \mathbb{R}$,

$$
\|u\|_{L_{t, x}^{3}\left(J \times \mathbb{R}^{5}\right)}<C\left(J,\left\|\left(u_{0}, u_{1}\right)\right\|_{\dot{B}_{1,1}^{3} \times \dot{B}_{1,1}^{2}}, \delta_{1}\right) .
$$

Proof. By Lemma 2.8, it suffices to show (3.59). And by the time reversibility of the wave equation, we just need consider the part of $t \geq 0$.

For $t \geq \delta_{1}$, by $u(t)=w(t)+v(t)$ and the formula (3.36) of $v(t)$, we have

$$
w_{t t}-\Delta w=-|u| u \text {. }
$$

We define the energy of $w$ as (1.2) by

$$
E(w(t))=\frac{1}{2} \int_{\mathbb{R}^{5}}\left|\nabla_{t} w(t)\right|^{2} d x+\frac{1}{2} \int_{\mathbb{R}^{5}}\left|\nabla_{x} w(t)\right|^{2} d x+\frac{1}{3} \int_{\mathbb{R}^{5}}|w(t)|^{3} d x .
$$

By the estimates (3.22), (3.38), Lemma 3.5 and interpolation, we have

$$
E\left(w\left(\delta_{1}\right)\right) \lesssim 1
$$

Now, we consider

$$
\begin{aligned}
& \left|\frac{d}{d t} E(w(t))\right| \\
= & \left|\int_{\mathbb{R}^{5}}(|w| w-|u| u) w_{t} d x\right| \\
\lesssim & \|v\|_{L_{x}^{6}\left(\mathbb{R}^{5}\right)}\left\|w_{t}\right\|_{L_{x}^{2}\left(\mathbb{R}^{5}\right)}\|w\|_{L_{x}^{3}\left(\mathbb{R}^{5}\right)}+\left\|w_{t}\right\|_{L_{x}^{2}\left(\mathbb{R}^{5}\right)}\|v\|_{L_{x}^{6}\left(\mathbb{R}^{5}\right)}\|v\|_{L_{x}^{3}\left(\mathbb{R}^{5}\right)} .
\end{aligned}
$$

By interpolation and the dispersive estimate (3.37) of $v$, we have

$$
\begin{aligned}
\|v\|_{L_{x}^{6}\left(\mathbb{R}^{5}\right)}\left\|w_{t}\right\|_{L_{x}^{2}\left(\mathbb{R}^{5}\right)}\|w\|_{L^{3}\left(\mathbb{R}^{5}\right)} & \lesssim \frac{1}{t}\|v\|_{L_{x}^{3}\left(\mathbb{R}^{5}\right)}^{\frac{1}{2}}\left\|w_{t}\right\|_{L_{x}^{2}\left(\mathbb{R}^{5}\right)}\|w\|_{L_{x}^{3}\left(\mathbb{R}^{5}\right)} \\
& \lesssim \frac{1}{t} E(w(t))^{\frac{5}{6}}\|v\|_{L_{x}^{3}\left(\mathbb{R}^{5}\right)}^{\frac{1}{2}} \lesssim \frac{1}{t}\left[E(w)(t)+\|v\|_{L_{x}^{3}\left(\mathbb{R}^{5}\right)}^{3}\right], \\
\left\|w_{t}\right\|_{L_{x}^{2}\left(\mathbb{R}^{5}\right)}\|v\|_{L_{x}^{6}\left(\mathbb{R}^{5}\right)}\|v\|_{L_{x}^{3}\left(\mathbb{R}^{5}\right)} & \lesssim \frac{1}{t} E(w(t))^{\frac{1}{2}}\|v\|_{L_{x}^{3}\left(\mathbb{R}^{5}\right)}^{\frac{3}{2}} \lesssim \frac{1}{t}\left[E(w)(t)+\|v\|_{L_{x}^{3}\left(\mathbb{R}^{5}\right)}^{3}\right] .
\end{aligned}
$$

Substituting (3.64) and (3.65) into (3.63), we obtain

$$
\frac{d}{d t} E(w(t)) \leq C \frac{1}{t}\left(E(w(t))+\|v\|_{L_{x}^{3}\left(\mathbb{R}^{5}\right)}^{3}\right) .
$$

Hence,

$$
\frac{d}{d t}\left[t^{-C}(E(w(t)))\right] \leq t^{-C-1}\|v\|_{L_{x}^{3}\left(\mathbb{R}^{5}\right)}^{3} .
$$

This estimate and the inequality (3.38) imply that

$$
E(w(t)) \leq C_{1}(1+|t|)^{C_{2}} .
$$


Thus, for any compact interval $J \subset \mathbb{R}$, we have

$$
\|u\|_{L_{t, x}^{3}\left(J \times \mathbb{R}^{5}\right)} \leq\|v\|_{L_{t, x}^{3}\left(J \times \mathbb{R}^{5}\right)}+\|w\|_{L_{t, x}^{3}\left(J \times \mathbb{R}^{5}\right)}<\infty .
$$

\section{SCATTERING}

In this section we prove the Theorem 1 by assuming Proposition 1.2, that is, removing the dependence of $\delta_{1}$ in (1.8). From the arguments in Section 3, we have $\delta_{1}=\frac{\delta}{2 R}$, where $\delta$ and $R$ depending the scaling and spatial profile of the initial data, respectively. We give the heuristic idea of the proof by analysing the effect of the parameters $\delta$ and $R$ on the critical norm $L_{t, x}^{3}\left(\mathbb{R} \times \mathbb{R}^{5}\right)$.

Note that the critical norm $L_{t, x}^{3}\left(\mathbb{R} \times \mathbb{R}^{5}\right)$ of the solution to the nonlinear equation (1.5) is invariant under the scaling transform. Hence the parameter $\delta$ may not be the main difficulty to prove Theorem 1. On the other hand, the latter parameter $R$ relies on the spatial profile of the initial data. For example, let $R$ be the parameter corresponding to the initial data $\left(u_{0}, u_{1}\right)$ with compact support. The linear evolution $\vec{S}(t)$ for $t \in \mathbb{R}$ does not change the critical norm $\dot{H}^{1 / 2} \times \dot{H}^{-1 / 2}$ by the Plancherel theorem, but owing to the Huygens principle for the odd-dimension linear wave equations, it does change the spatial support of the initial datum. Thus, for the initial data $\vec{S}\left(t_{0}\right)\left(u_{0}, u_{1}\right)$, the spatial parameter(may be chosen as $R+t_{0}$ ) is likely very huge, when $t_{0}$ is large enough. However, the $\dot{B}_{1,1}^{3} \times \dot{B}_{1,1}^{2}$ norm may become huge under the evolution of $\vec{S}(t)$. Indeed, if $\left\|\left(u_{0}, u_{1}\right)\right\|_{\dot{B}_{1,1}^{3} \times \dot{B}_{1,1}^{2}\left(\mathbb{R}^{5}\right)}=1$, then we have $\left\|\vec{S}\left(t_{0}\right)\left(u_{0}, u_{1}\right)\right\|_{\dot{B}_{1,1}^{3} \times \dot{B}_{1,1}^{2}\left(\mathbb{R}^{5}\right)} \rightarrow \infty$ as $t_{0} \rightarrow \infty$. 1 Hence, if $\left\|\vec{S}\left(t_{0}\right)\left(u_{0}, u_{1}\right)\right\|_{\dot{B}_{1,1}^{3} \times \dot{B}_{1,1}^{2}\left(\mathbb{R}^{5}\right)} \lesssim 1$, then $t_{0}$ remains bounded. Taking account of this fact, one may conquer the difficulties caused by the parameter $R$.

To finish the proof of Theorem 1, we need the following theorem of profile decomposition.

4.1. Profile decomposition. Now, we recall the linear profile decomposition from [24] in the radial case. We refer to [1] for the profile decompositions in the energy critical spaces.

Theorem 5 (Profile decomposition). Let $C>0$ be a fixed number and let $\left(u_{0}^{n}, u_{1}^{n}\right)_{n}$ be a sequence in $\dot{H}^{1 / 2} \times \dot{H}^{-1 / 2}\left(\mathbb{R}^{5}\right)$, with

$$
\left\|\left(u_{0}^{n}, u_{1}^{n}\right)\right\|_{\dot{H}^{1 / 2} \times \dot{H}^{-1 / 2}\left(\mathbb{R}^{5}\right)} \leq C .
$$

Then there exist a subsequence of $\left(u_{0}^{n}, u_{1}^{n}\right)$ (which we still denote by $\left(u_{0}^{n}, u_{1}^{n}\right)$ ), a sequence $\left(\phi_{0}^{j}, \phi_{1}^{j}\right)_{j \in \mathbb{N}} \subset$ $\dot{H}^{1 / 2} \times \dot{H}^{-1 / 2}\left(\mathbb{R}^{5}\right)$, a sequence $\left(R_{0, n}^{N}, R_{1, n}^{N}\right)_{N \geq 1} \subset \dot{H}^{1 / 2} \times \dot{H}^{-1 / 2}\left(\mathbb{R}^{5}\right)$ and a sequence of parameters $\left(t_{j}^{n}, \lambda_{j}^{n}\right) \subset \mathbb{R} \times(0, \infty)$ such that for each $N \geq 1$,

$$
S(t)\left(u_{0}^{n}, u_{1}^{n}\right)=\sum_{j=1}^{N}\left(\lambda_{j}^{n}\right)^{2}\left[S\left(\lambda_{j}^{n}\left(t-t_{j}^{n}\right)\right)\left(\phi_{0}^{j}, \phi_{1}^{j}\right)\right]\left(\lambda_{j}^{n} x\right)+S(t)\left(R_{0, n}^{N}, R_{1, n}^{N}\right)
$$

with

$$
\lim _{N \rightarrow \infty} \limsup _{n \rightarrow \infty}\left\|S(t)\left(R_{0, n}^{N}, R_{1, n}^{N}\right)\right\|_{L_{t, x}^{3}\left(\mathbb{R} \times \mathbb{R}^{5}\right)}=0 .
$$

In addition, the parameters $\left(t_{j}^{n}, \lambda_{j}^{n}\right)$ satisfy the orthogonality property: for any $j \neq k$,

$$
\lim _{n \rightarrow \infty}\left(\frac{\lambda_{j}^{n}}{\lambda_{k}^{n}}+\frac{\lambda_{k}^{n}}{\lambda_{j}^{n}}+\left(\lambda_{k}^{n}\right)^{\frac{1}{2}}\left(\lambda_{j}^{n}\right)^{\frac{1}{2}}\left|t_{j}^{n}-t_{k}^{n}\right|\right)=\infty .
$$

\footnotetext{
${ }^{1}$ By interpolation and density, it suffices to show that $\lim _{t \rightarrow \infty}\left\|e^{i t|\nabla|} f\right\|_{\dot{B}_{\infty}^{-2}, \infty\left(\mathbb{R}^{5}\right)}=0$, for $f \in \mathcal{S}\left(\mathbb{R}^{5}\right)$, which is a direct consequence of the dispersive estimate (2.20) and Bernstein's estimates.
} 
Furthermore, for every $N \geq 1$,

$$
\left\|\left(u_{0}^{n}, u_{1}^{n}\right)\right\|_{\dot{H}^{1 / 2} \times \dot{H}^{-1 / 2}\left(\mathbb{R}^{5}\right)}^{2}=\sum_{j=1}^{N}\left\|\left(\phi_{0}^{j}, \phi_{1}^{j}\right)\right\|_{\dot{H}^{1 / 2} \times \dot{H}^{-1 / 2}\left(\mathbb{R}^{5}\right)}^{2}+\left\|\left(R_{0, n}^{N}, R_{1, n}^{N}\right)\right\|_{\dot{H}^{1 / 2} \times \dot{H}^{-1 / 2}\left(\mathbb{R}^{5}\right)}^{2}+o_{n}(1) .
$$

4.2. End the proof of the main theorem. Now, we apply the strategy in [6] to finish the proof of Theorem 1, that is, remove the $\delta_{1}$ in Proposition 1.2,

We prove the Theorem 1 by contradiction. We assume $u$ is the solution to (1.5) with the initial data such that $\left(u_{0}, u_{1}\right) \in \dot{B}_{1,1}^{3} \times \dot{B}_{1,1}^{2}\left(\mathbb{R}^{5}\right)$. For $M \geq 0$, let

$$
f(M)=\sup \left\{\|u\|_{L_{t, x}^{3}\left(\mathbb{R} \times \mathbb{R}^{5}\right)}:\left\|\left(u_{0}, u_{1}\right)\right\|_{\dot{B}_{1,1}^{3} \times \dot{B}_{1,1}^{2}\left(\mathbb{R}^{5}\right)} \leq M\right\} .
$$

Then by Proposition 1.2, $f$ is well defined. In addition, by Bernstein and Theorem 2, $f(M)<\infty$ when $M$ is small enough. From the definition, $f(M)$ is non-decreasing as $M$ increases.

If Theorem 1 fails, then there exist $M_{0}<\infty$ and a sequence $\left\{\left(u_{0}^{n}, u_{1}^{n}\right)\right\}_{n \in \mathbb{N}} \subset \dot{B}_{1,1}^{3} \times \dot{B}_{1,1}^{2}\left(\mathbb{R}^{5}\right)$, such that

$$
\left\|\left(u_{0}^{n}, u_{1}^{n}\right)\right\|_{\dot{B}_{1,1}^{3} \times \dot{B}_{1,1}^{2}\left(\mathbb{R}^{5}\right)} \leq M_{0}
$$

and the solution $u^{n}$ to (1.5) with $\left(u^{n}(0),\left(\partial_{t} u^{n}\right)(0)\right)=\left(u_{0}^{n}, u_{1}^{n}\right)$ satisfying

$$
\left\|u^{n}\right\|_{L_{t, x}^{3}\left(\mathbb{R} \times \mathbb{R}^{5}\right)} \rightarrow \infty
$$

as $n \rightarrow \infty$. By Theorem 5, we have

$$
S(t)\left(u_{0}^{n}, u_{1}^{n}\right)=\sum_{j=1}^{N}\left(\lambda_{j}^{n}\right)^{2}\left[S\left(\lambda_{j}^{n}\left(t-t_{j}^{n}\right)\right)\left(\phi_{0}^{j}, \phi_{1}^{j}\right)\right]\left(\lambda_{j}^{n} x\right)+S(t)\left(R_{0, n}^{N}, R_{1, n}^{N}\right) .
$$

In the proof of Theorem 5, the author in [24] actually proved that

$$
\left.\vec{S}\left(t+t_{j}^{n} \lambda_{j}^{n}\right)\left[\left(\lambda_{j}^{n}\right)^{-2} u_{0}^{n}\left(\frac{\cdot}{\lambda_{j}^{n}}\right),\left(\lambda_{j}^{n}\right)^{-3} u_{1}^{n}\left(\frac{\cdot}{\lambda_{j}^{n}}\right)\right]\right|_{t=0} \rightarrow\left(\phi_{0}^{j}, \phi_{1}^{j}\right),
$$

weakly in $\dot{H}^{1 / 2} \times \dot{H}^{-1 / 2}\left(\mathbb{R}^{5}\right)$ as $n \rightarrow \infty$. From this we can prove the following proposition.

Proposition 4.1. For fixed $j \in \mathbb{N}$, if $\left(\phi_{0}^{j}, \phi_{1}^{j}\right) \neq 0$, then $\left|t_{j}^{n} \lambda_{j}^{n}\right|$ is bounded as $n \rightarrow \infty$.

Proof. First, if $t_{j}^{n} \lambda_{j}^{n}$ is unbounded for $n \in \mathbb{N}$, then by taking a subsequence of $n$ (still denoted by $\left.n\right)$, we assume that $\left|t_{j}^{n} \lambda_{j}^{n}\right| \rightarrow \infty$, as $n \rightarrow \infty$. In light of the heuristic analysis at the beginning of this section, we may have

$$
\vec{S}\left(t_{j}^{n} \lambda_{j}^{n}\right)\left[\left(\lambda_{j}^{n}\right)^{-2} u_{0}^{n}\left(\frac{\cdot}{\lambda_{j}^{n}}\right),\left(\lambda_{j}^{n}\right)^{-3} u_{1}^{n}\left(\frac{\cdot}{\lambda_{j}^{n}}\right)\right] \rightarrow(0,0) .
$$

in $L_{x}^{3} \times W_{x}^{-1,3}\left(\mathbb{R}^{5}\right)$. In fact, using (4.7) and the estimate (3.30) in Section 3, we have

$$
\begin{aligned}
&\left\|S\left(t_{j}^{n} \lambda_{j}^{n}\right)\left[\left(\lambda_{j}^{n}\right)^{-2} u_{0}^{n}\left(\frac{\cdot}{\lambda_{j}^{n}}\right),\left(\lambda_{j}^{n}\right)^{-3} u_{1}^{n}\left(\frac{\cdot}{\lambda_{j}^{n}}\right)\right]\right\|_{L_{x}^{3}\left(\mathbb{R}^{5}\right)} \\
& \lesssim\left|t_{j}^{n} \lambda_{j}^{n}\right|^{-\frac{1}{3}}\left\|\left(\left(\lambda_{j}^{n}\right)^{-2} u_{0}^{n}\left(\frac{\cdot}{\lambda_{j}^{n}}\right),\left(\lambda_{j}^{n}\right)^{-3} u_{1}^{n}\left(\frac{\cdot}{\lambda_{j}^{n}}\right)\right)\right\|_{\dot{B}_{1,1}^{3} \times \dot{B}_{1,1}^{2}\left(\mathbb{R}^{5}\right)} \\
& \lesssim\left|t_{j}^{n} \lambda_{j}^{n}\right|^{-\frac{1}{3}}\left\|\left(u_{0}^{n}, u_{1}^{n}\right)\right\|_{\dot{B}_{1,1}^{3} \times \dot{B}_{1,1}^{2}\left(\mathbb{R}^{5}\right)} \rightarrow 0,
\end{aligned}
$$


as $n \rightarrow \infty$. Similarly, by the dispersive estimate (2.20), Bernstein and interpolation, we have

$$
\begin{aligned}
& \left\|\dot{S}\left(t_{j}^{n} \lambda_{j}^{n}\right)\left[\left(\lambda_{j}^{n}\right)^{-2} u_{0}^{n}\left(\frac{\cdot}{\lambda_{j}^{n}}\right),\left(\lambda_{j}^{n}\right)^{-3} u_{1}^{n}\left(\frac{\dot{\lambda_{j}^{n}}}{j}\right)\right]\right\|_{\dot{W}_{x}^{-1,3}\left(\mathbb{R}^{5}\right)} \\
\lesssim & \left|t_{j}^{n} \lambda_{j}^{n}\right|^{-\frac{1}{3}}\left\|\left(u_{0}^{n}, u_{1}^{n}\right)\right\|_{\dot{B}_{1,1}^{3} \times \dot{B}_{1,1}^{2}\left(\mathbb{R}^{5}\right)} \rightarrow 0,
\end{aligned}
$$

as $n \rightarrow \infty$. Hence, from the weak convergence relation (4.10), (4.12) and (4.13) imply that $\left(\phi_{0}^{j}, \phi_{1}^{j}\right)=$ $(0,0)$.

For simplicity, we assume that every $\left(\phi_{0}^{j}, \phi_{1}^{j}\right)$ in (4.9) is nontrivial. By Proposition 4.1, for each fixed $j, t_{j}^{n} \lambda_{j}^{n}$ is bounded, and therefore after taking a suitable subsequence of $n$ (still denoted by $n$ ), we can assume $t_{j}^{n} \lambda_{j}^{n} \rightarrow t_{j} \in \mathbb{R}$ as $n \rightarrow \infty$. Hence, if we denote $\left(\varphi_{0}^{j}, \varphi_{1}^{j}\right)=\vec{S}\left(-t_{j}\right)\left(\phi_{0}^{j}, \phi_{1}^{j}\right)$, then

$$
\vec{S}\left(-\lambda_{j}^{n} t_{j}^{n}\right)\left(\phi_{0}^{j}, \phi_{1}^{j}\right)-\left(\varphi_{0}^{j}, \varphi_{1}^{j}\right) \rightarrow 0,
$$

in $\dot{H}^{1 / 2} \times \dot{H}^{-1 / 2}\left(\mathbb{R}^{5}\right)$ as $n \rightarrow \infty$. Let

$$
\left\{\begin{array}{l}
\tilde{R}_{0, n}^{N}=R_{0, n}^{N}+\sum_{j=1}^{N}\left(\lambda_{j}^{n}\right)^{2}\left[S\left(-\lambda_{j}^{n} t_{j}^{n}\right)\left(\phi_{0}^{j}, \phi_{1}^{j}\right)\right]\left(\lambda_{j}^{n} x\right)-\left(\lambda_{j}^{n}\right)^{2}\left(\varphi_{0}^{j}, \varphi_{1}^{j}\right)\left(\lambda_{j}^{n} x\right), \\
\tilde{R}_{1, n}^{N}=R_{1, n}^{N}+\sum_{j=1}^{N}\left(\lambda_{j}^{n}\right)^{3}\left[\dot{S}\left(-\lambda_{j}^{n} t_{j}^{n}\right)\left(\phi_{0}^{j}, \phi_{1}^{j}\right)\right]\left(\lambda_{j}^{n} x\right)-\left(\lambda_{j}^{n}\right)^{3}\left[( \varphi _ { 0 } ^ { j } , \varphi _ { 1 } ^ { j } ) \left[\left(\lambda_{j}^{n} x\right),\right.\right.
\end{array}\right.
$$

then

$$
\lim _{N \rightarrow \infty} \limsup _{n \rightarrow \infty}\left\|S(t)\left(\tilde{R}_{0, n}^{N}, \tilde{R}_{1, n}^{N}\right)\right\|_{L_{t, x}^{3}\left(\mathbb{R} \times \mathbb{R}^{5}\right)}=0 .
$$

Taking $t=0$ in (4.9), by (4.14) and (4.15), we have

$$
\left(u_{0}^{n}, u_{1}^{n}\right)=\sum_{j=1}^{N}\left(\left(\lambda_{j}^{n}\right)^{2}\left(\varphi_{0}^{j}\left(\lambda_{j}^{n} x\right),\left(\lambda_{j}^{n}\right)^{3} \varphi_{1}^{j}\left(\lambda_{j}^{n} x\right)\right)+\left(\tilde{R}_{0, n}^{N}, \tilde{R}_{1, n}^{N}\right) .\right.
$$

In addition, by the orthogonality (4.4) and Proposition 4.1, we have for each $j \neq k$

$$
\lim _{n \rightarrow \infty} \frac{\lambda_{j}^{n}}{\lambda_{k}^{n}}+\frac{\lambda_{k}^{n}}{\lambda_{j}^{n}}=\infty
$$

as $n \rightarrow \infty$. Thus, for fixed $j \in \mathbb{N}$, we have

$$
\left(\left(\lambda_{j}^{n}\right)^{-2} u_{0}^{n}\left(\frac{x}{\lambda_{j}^{n}}\right),\left(\lambda_{j}^{n}\right)^{-3} u_{1}^{n}\left(\frac{x}{\lambda_{j}^{n}}\right)\right) \rightarrow\left(\varphi_{0}^{j}, \varphi_{1}^{j}\right)
$$

weakly in $\dot{H}^{1 / 2} \times \dot{H}^{-1 / 2}\left(\mathbb{R}^{5}\right)$ as $n \rightarrow \infty$. By Fatou's lemma, this fact and the inequality (4.7) imply

$$
\left\|\left(\varphi_{0}^{j}, \varphi_{1}^{j}\right)\right\|_{\dot{B}_{1,1}^{3} \times \dot{B}_{1,1}^{2}\left(\mathbb{R}^{5}\right)} \leq M_{0}
$$

On the other hand, (4.5) and (4.14) yield that

$$
\sum_{j \geq 1}\left\|\left(\varphi_{0}^{j}, \varphi_{1}^{j}\right)\right\|_{\dot{H}^{1 / 2} \times \dot{H}^{-1 / 2}\left(\mathbb{R}^{5}\right)}^{2} \lesssim \sup _{n \geq 1}\left\|\left(u_{0}^{n}, u_{1}^{n}\right)\right\|_{\dot{H}^{1 / 2} \times \dot{H}^{-1 / 2}\left(\mathbb{R}^{5}\right)}^{2} \lesssim C_{0}^{2} .
$$

Hence, for fixed $\epsilon>0$, there exists a finite integer $N_{0}$ such that

$$
\sum_{j \geq N_{0}+1}\left\|\left(\varphi_{0}^{j}, \varphi_{1}^{j}\right)\right\|_{\dot{H}^{1 / 2} \times \dot{H}^{-1 / 2}\left(\mathbb{R}^{5}\right)}^{2} \leq \epsilon
$$


By the local well-posedness theory, if $\epsilon>0$ is small enough, then the solution $v^{j}$ to (1.5) with the initial data $\left(\varphi_{0}^{j}, \varphi_{1}^{j}\right)$ is globally well-posed and

$$
\left\|v^{j}\right\|_{L_{t, x}^{3}\left(\mathbb{R} \times \mathbb{R}^{5}\right)} \lesssim\left\|\left(\varphi_{0}^{j}, \varphi_{1}^{j}\right)\right\|_{\dot{H}^{1 / 2} \times \dot{H}^{-1 / 2}\left(\mathbb{R}^{5}\right)}, \text { for every } j \geq N_{0}+1 .
$$

For $1 \leq j \leq N_{0}$, as a consequence of Proposition 1.2, the solution to (1.5) with the initial data $\left(\varphi_{0}^{j}, \varphi_{1}^{j}\right)$ is globally well-posed and such that

$$
\left\|v^{j}\right\|_{L_{t, x}^{3}\left(\mathbb{R} \times \mathbb{R}^{5}\right)} \lesssim M_{0, j} 1
$$

By the orthogonality property (4.18), for any $j \neq k$

$$
\lim _{n \rightarrow \infty} \iint_{\mathbb{R} \times \mathbb{R}^{5}}\left|\left(\lambda_{j}^{n}\right)^{2} v^{j}\left(\lambda_{j}^{n} t, \lambda_{j}^{n} x\right)\right|^{2}\left|\left(\lambda_{k}^{n}\right)^{2} v^{k}\left(\lambda_{k}^{n} t, \lambda_{k}^{n} x\right)\right| d x d t=0 .
$$

This together with the estimates (4.22), (4.23) and (4.24) implies

$$
\sup _{N \geq N_{0}+1} \lim _{n \rightarrow \infty}\left\|\sum_{1 \leq j \leq N}\left(\lambda_{j}^{n}\right)^{2} v^{j}\left(\lambda_{j}^{n} t, \lambda_{j}^{n} x\right)\right\|_{L_{t, x}^{3}\left(\mathbb{R} \times \mathbb{R}^{5}\right)}
$$

is bounded. Similarly, as a consequence of the trivial estimate

$$
\begin{aligned}
& \left.\lesssim \sum_{j=1}^{N}\left(\lambda_{j}^{n}\right)^{2} v^{j}\left(\lambda_{j}^{n} t, \lambda_{j}^{n} x\right)\right)-\sum_{j=1}^{N} F\left(\left(\lambda_{j}^{n}\right)^{2} v^{j}\left(\lambda_{j}^{n} t, \lambda_{j}^{n} x\right)\right) \mid \\
& \left.\mid\left(\lambda_{j}^{n}\right)^{2} v^{j}\left(\lambda_{j}^{n} t, \lambda_{j}^{n} x\right)\right)\left|\left(\lambda_{k}^{n}\right)^{2} v^{k}\left(\lambda_{k}^{n} t, \lambda_{k}^{n} x\right)\right|
\end{aligned}
$$

and the orthogonality property (4.18), we have

$$
\lim _{n \rightarrow \infty}\left\|F\left(\sum_{j=1}^{N}\left(\lambda_{j}^{n}\right)^{2} v^{j}\left(\lambda_{j}^{n} t, \lambda_{j}^{n} x\right)\right)-\sum_{j=1}^{N} F\left(\left(\lambda_{j}^{n}\right)^{2} v^{j}\left(\lambda_{j}^{n} t, \lambda_{j}^{n} x\right)\right)\right\|_{L_{t, x}^{\frac{3}{2}}\left(\mathbb{R} \times \mathbb{R}^{5}\right)}=0 .
$$

Let $u_{N}^{n}$ be an approximate solution to (1.5) defined by

$$
u_{N}^{n}=\sum_{j=1}^{N}\left(\lambda_{j}^{n}\right)^{2} v^{j}\left(\lambda_{j}^{n} x\right)+S(t)\left(\tilde{R}_{0, n}^{N}, \tilde{R}_{1, n}^{N}\right) .
$$

Then, recall the property (4.16) for $\left(\tilde{R}_{0, n}^{N}, \tilde{R}_{1, n}^{N}\right)$ and the fact that (4.26) is uniformly bounded for $N \geq N_{0}+1$, we obtain

$$
\limsup _{N \rightarrow \infty} \lim _{n \rightarrow \infty}\left\|u_{N}^{n}\right\|_{L_{t, x}^{3}\left(\mathbb{R} \times \mathbb{R}^{5}\right)} \lesssim 1
$$

Moreover, combining (4.27), the property (4.16) for $\left(\tilde{R}_{0, n}^{N}, \tilde{R}_{1, n}^{N}\right)$, and Hölder's inequality, we have

$$
\limsup _{N \rightarrow \infty} \lim _{n \rightarrow \infty}\left\|F\left(u_{N}^{n}\right)-\sum_{j=1}^{N} F\left(\left(\lambda_{j}^{n}\right)^{2} v^{j}\left(\lambda_{j}^{n} t, \lambda_{j}^{n} x\right)\right)\right\|_{L_{t, x}^{\frac{3}{2}}\left(\mathbb{R} \times \mathbb{R}^{5}\right)}=0 .
$$

Utilizing Theorem 3 , by (4.17), (4.28) and (4.29), we have that for $n$ large enough, the solution $u^{n}$ to the equation (1.5) with initial data $\left(u_{0}^{n}, u_{1}^{n}\right)$ is global and such that

$$
\lim _{n \rightarrow \infty}\left\|u^{n}\right\|_{L_{t, x}^{3}\left(\mathbb{R} \times \mathbb{R}^{5}\right)}
$$

is bounded, which contradicts with the hypothesis (4.8) of $u^{n}$. Thus, we have proved Theorem 1, 


\section{Hyperbolic Coordinates and spacetime estimates}

In this section, we will finish the proof of Proposition [1.2. We first reduce Proposition 1.2 to estimating the $L_{t, x}^{3}$ norm of $w$ on the region $\Omega_{2}$, which will be defined below. Without loss of generality, we assume that $\delta_{1}<\frac{1}{4}$. And as in Subsection 4, we also note the constants in " $\lesssim$ " in the following of this section may be different in each step and are dependent on $\delta_{1}$ and $\left\|\left(u_{0}, u_{1}\right)\right\|_{\dot{B}_{1,1}^{3} \times \dot{B}_{1,1}^{2}\left(\mathbb{R}^{5}\right)}$.

5.1. Reduction of the proof of Proposition 1.2. Now we consider the $L_{t, x}^{3}$ norm of $u$ on the $\mathbb{R}_{+} \times \mathbb{R}^{5}$. First, we split time-spatial region $\mathbb{R}_{+} \times \mathbb{R}^{5}$ as the union

$$
\mathbb{R}_{+} \times \mathbb{R}^{5}=\Omega_{1} \cup \Omega_{2} \cup \Omega_{3},
$$

where $\Omega_{1}=\left\{(t, x) \in \mathbb{R}_{+} \times \mathbb{R}^{5}:|x| \geq t+\frac{1}{2}\right\}$ and $\Omega_{2}=\left\{(t, x) \in \mathbb{R}_{+} \times \mathbb{R}^{5}:\left(t+\left(1-\delta_{1}\right)\right)^{2}-|x|^{2} \geq 1\right\}$. Since $\delta_{1}<\frac{1}{4}$, there exists a large constant $C>0$, such that

$$
\Omega_{3} \subset\left\{(t, x) \in \mathbb{R}_{+} \times \mathbb{R}^{5}: t+|x| \leq C\right\} .
$$

Recalling the estimate (3.23) in Section 3, we obtain $\|u\|_{L_{t, x}^{3}\left(\Omega_{1}\right)} \lesssim 1$. For the bounded region $\Omega_{3}$, Theorem 4 yields $\|u\|_{L_{t, x}^{3}\left(\Omega_{3}\right)} \lesssim 1$. Hence, we just need to consider the $L_{t, x}^{3}$ norm of $u$ on the region $\Omega_{2}$. By the estimate (3.38) for $v$, we are reduced to show $\|w\|_{L_{t, x}^{3}\left(\Omega_{2}\right)} \lesssim 1$.

5.2. Hyperbolic coordinates. For the radial solution $u(t, x)$ to (1.5), if we denote $u(t, r)=u(t, x)$ for $r=|x|$, then

$$
\partial_{t t}\left(r^{2} u\right)-\partial_{r r}\left(r^{2} u\right)=-2 u-r^{2}|u| u .
$$

Denote $\bar{u}(t, r)=u\left(t-\left(1-\delta_{1}\right), r\right)$ and $\bar{v}, \bar{w}$ similarly. Let $(t, r)=\left(e^{\tau} \cosh s, e^{\tau} \sinh s\right)$, then $d r d t=$ $e^{2 \tau} d \tau d s$. We denote the hyperbolic transforms by

$$
\begin{aligned}
& \tilde{u}=\frac{e^{2 \tau} \sinh ^{2} s}{s^{2}} \bar{u}\left(e^{\tau} \cosh s, e^{\tau} \sinh s\right), \\
& \tilde{v}=\frac{e^{2 \tau} \sinh ^{2} s}{s^{2}} \bar{v}\left(e^{\tau} \cosh s, e^{\tau} \sinh s\right),
\end{aligned}
$$

and

$$
\tilde{w}=\frac{e^{2 \tau} \sinh ^{2} s}{s^{2}} \bar{w}\left(e^{\tau} \cosh s, e^{\tau} \sinh s\right) .
$$

Hence, we have

$$
\begin{gathered}
\partial_{\tau \tau}\left(s^{2} \tilde{u}\right)-\partial_{s s}\left(s^{2} \tilde{u}\right)=-\frac{2 s^{2}}{\sinh ^{2} s} \tilde{u}-\frac{s^{4}}{\sinh ^{2} s}|\tilde{u}| \tilde{u}, \\
\partial_{\tau \tau}\left(s^{2} \tilde{v}\right)-\partial_{s s}\left(s^{2} \tilde{v}\right)=-\frac{2 s^{2}}{\sinh ^{2} s} \tilde{v}, \\
\partial_{\tau \tau}\left(s^{2} \tilde{w}\right)-\partial_{s s}\left(s^{2} \tilde{w}\right)=-\frac{2 s^{2}}{\sinh ^{2} s} \tilde{w}-\frac{s^{4}}{\sinh ^{2} s}|\tilde{u}| \tilde{u} .
\end{gathered}
$$

Define the hyperbolic energy of $\tilde{w}$ by

$$
E_{h}(\tilde{w})(\tau)=\int_{0}^{\infty}\left[\frac{1}{2}\left|\left(s^{2} \tilde{w}\right)_{\tau}\right|^{2}+\frac{1}{2}\left|\left(s^{2} \tilde{w}\right)_{s}\right|^{2}+\frac{\left|s^{2} \tilde{w}\right|^{2}}{\sinh ^{2} s}+\frac{1}{3} \frac{\left|s^{2} \tilde{w}\right|^{3}}{\sinh ^{2} s}\right] d s .
$$


5.3. The hyperbolic energy for some $\tau_{0} \geq 0$. First, we want to prove $E_{h}(\tilde{w})(\tau)$ is bounded for some $\tau_{0} \geq 0$. We claim that it suffices to show the boundedness of

$$
\int_{0}^{\infty}\left[\left|\left(s^{2} \tilde{w}\right)_{\tau}\left(\tau_{0}, s\right)\right|^{2}+\left|\left(s^{2} \tilde{w}\right)_{s}\left(\tau_{0}, s\right)\right|^{2}\right] d s
$$

for some $\tau_{0}>0$.

To prove this claim, we need the sharp Hardy inequality,

$$
\left(\frac{d-2}{2}\right)^{2} \int_{\mathbb{R}^{d}} \frac{|f(x)|^{2}}{|x|^{2}} d x \leq \int_{\mathbb{R}^{d}}|\nabla f|^{2}(x) d x .
$$

By polar coordinates, we rewrite this inequality for radial functions,

$$
\left(\frac{d-2}{2}\right)^{2} \int_{0}^{\infty}|f(r)|^{2} r^{d-3} d r \leq \int_{0}^{\infty}\left|\partial_{r} f(r)\right|^{2}(r) r^{d-1} d r
$$

Then, this inequality and integration by parts imply that

$$
\begin{aligned}
\int_{0}^{\infty}\left|\left(s^{2} \tilde{w}\left(\tau_{0}\right)\right)_{s}\right|^{2} d s & =\int_{0}^{\infty} s^{4} \tilde{w}_{s}^{2}\left(\tau_{0}\right) d s+4 \int_{0}^{\infty} s^{2} \tilde{w}\left(\tau_{0}\right) s \tilde{w}_{s}\left(\tau_{0}\right) d s+4 \int_{0}^{\infty} s^{2} \tilde{w}^{2}\left(\tau_{0}\right) d s \\
& =\int_{0}^{\infty} s^{4} \tilde{w}_{s}^{2}\left(\tau_{0}\right) d s-2 \int_{0}^{\infty} s^{2} \tilde{w}\left(\tau_{0}\right)^{2} d s \geq \frac{1}{9} \int_{0}^{\infty} s^{4} \tilde{w}_{s}^{2}\left(\tau_{0}\right) d s .
\end{aligned}
$$

In addition, by Hölder and Sobolev in polar coordinates, we have

$$
\int_{0}^{\infty} \frac{\left|s^{2} \tilde{w}\left(\tau_{0}\right)\right|^{3}}{\sinh ^{2} s} d s=\int_{0}^{\infty} \frac{s^{2}}{\sinh ^{2} s}\left|\tilde{w}\left(\tau_{0}\right)\right|^{3} s^{4} d s \lesssim\left(\int_{0}^{\infty}\left|\tilde{w}\left(\tau_{0}\right)\right|^{\frac{10}{3}} s^{4} d s\right)^{\frac{9}{10}} \lesssim\left(\int_{0}^{\infty}\left|\tilde{w}_{s}\left(\tau_{0}\right)\right|^{2} s^{4} d s\right)^{\frac{3}{2}} .
$$

By Hardy's inequality, we have

$$
\int_{0}^{\infty} \frac{\left|s^{2} \tilde{w}\left(\tau_{0}\right)\right|^{2}}{\sinh ^{2} s} d s \lesssim \int_{0}^{\infty} \frac{1}{s^{2}}\left|\tilde{w}\left(\tau_{0}\right)\right|^{2} s^{4} d s \lesssim \int_{0}^{\infty}\left|\tilde{w}_{s}\left(\tau_{0}\right)\right|^{2} s^{4} d s .
$$

Hence the claim follows.

5.3.1. The hyperbolic energy for $s>s_{0}>0$. For $\tau \in[0,1]$ and sufficiently large $s_{0}>0$, we can assume that $e^{\tau-s_{0}}<\frac{1}{2}-\delta_{1}$. By the finite speed of propagation, $v(t, r)$ are supported in the region $\left\{(t, r) \in \mathbb{R} \times \mathbb{R}_{+}: r-t \lesssim \delta_{1} / 5\right\}$. Then, for $\tau \in[0,1]$ and $s \geq s_{0}$, we have

$$
e^{\tau} \sinh s-\left[e^{\tau} \cosh s-\left(1-\delta_{1}\right)\right]=1-\delta_{1}-e^{\tau-s}>\frac{1}{2}>\frac{\delta_{1}}{5},
$$

which leads to $v\left(e^{\tau} \cosh s-\left(1-\delta_{1}\right), e^{\tau} \sinh s\right)=0$. Hence, for $\tau \in[0,1]$, we have

$$
\int_{s_{0}}^{\infty} \frac{1}{2}\left|\left(s^{2} \tilde{w}\right)_{\tau}(\tau, s)\right|^{2}+\frac{1}{2}\left|\left(s^{2} \tilde{w}\right)_{s}(\tau, s)\right|^{2} d s=\int_{s_{0}}^{\infty} \frac{1}{2}\left|\left(s^{2} \tilde{u}\right)_{\tau}(\tau, s)\right|^{2}+\frac{1}{2}\left|\left(s^{2} \tilde{u}\right)_{s}(\tau, s)\right|^{2} d s .
$$

Since $u$ is a radial solution to the equation (1.5), we have, by (2.19),

$$
\begin{aligned}
r^{2} u(t, r)= & \frac{1}{2}\left[(r-t)^{2} u_{0}(r-t)+(r+t)^{2} u_{0}(r+t)\right] \\
& -\frac{1}{2} t r^{-1} \int_{r-t}^{r+t} s u_{0}(s) d s \\
& +\frac{1}{4 r} \int_{r-t}^{r+t} s\left(s^{2}+r^{2}-t^{2}\right) u_{1}(s) d s \\
& +\frac{1}{4 r} \int_{0}^{t} \int_{r-t+s}^{r+t-s} \rho\left(\rho^{2}+r^{2}-(t-s)^{2}\right)|u| u(s, \rho) d \rho d s
\end{aligned}
$$

for $r \geq t \geq 0$. Hence, by the hyperbolic transform (5.3), we have 


$$
\begin{aligned}
& s^{2} \tilde{u}(\tau, s) \\
= & \frac{1}{2}\left[\left(\left(1-\delta_{1}-e^{\tau-s}\right)^{2} u_{0}\left(1-\delta_{1}-e^{\tau-s}\right)+\left(e^{\tau+s}-\left(1-\delta_{1}\right)\right)^{2} u_{0}\left(e^{\tau+s}-\left(1-\delta_{1}\right)\right)\right]\right. \\
& -\frac{1}{2}\left(e^{\tau} \cosh s-\left(1-\delta_{1}\right)\right)\left(e^{\tau} \sinh s\right)^{-1} \int_{1-\delta_{1}-e^{\tau-s}}^{e^{\tau+s}-\left(1-\delta_{1}\right)} \rho u_{0}(\rho) d \rho \\
& +\frac{1}{4} \int_{1-\delta_{1}-e^{\tau-s}}^{e^{\tau+s}-\left(1-\delta_{1}\right)} \rho \frac{\rho^{2}+\left(e^{\tau+s}-\left(1-\delta_{1}\right)\right)\left(1-\delta_{1}-e^{\tau-s}\right)}{e^{\tau} \sinh s} u_{1}(\rho) d \rho \\
& +\frac{1}{4} \int_{1-\delta_{1}}^{e^{\tau} \cosh s} \int_{t-e^{\tau-s}}^{e^{\tau+s}-t} \rho \frac{\rho^{2}+\left(e^{\tau+s}-t\right)\left(t-e^{\tau-s}\right)}{e^{\tau} \sinh s}|\bar{u}| \bar{u}(t, \rho) d \rho d t .
\end{aligned}
$$

For (5.18), by a direct calculation, we obtain

$$
\begin{aligned}
& \left(\partial_{\tau}+\partial_{s}\right)(\underline{5.18)})=2\left(e^{\tau+s}-\left(1-\delta_{1}\right)\right) e^{\tau+s} u_{0}\left(e^{\tau+s}-\left(1-\delta_{1}\right)\right)+\left(e^{\tau+s}-\left(1-\delta_{1}\right)\right)^{2} u_{0}^{\prime}\left(e^{\tau+s}-\left(1-\delta_{1}\right)\right) e^{\tau+s}, \\
& \left(\partial_{\tau}-\partial_{s}\right)(\underline{5.18})=2\left(1-\delta_{1}-e^{\tau-s}\right) e^{\tau-s} u_{0}\left(1-\delta_{1}-e^{\tau-s}\right)+\left(\left(1-\delta_{1}-e^{\tau-s}\right)^{2} u_{0}^{\prime}\left(1-\delta_{1}-e^{\tau-s}\right) e^{\tau-s}\right.
\end{aligned}
$$

Using the estimate (2.13) in Section 2 and polar coordinates, we deduce that

$$
\begin{gathered}
\int_{s_{0}}\left|\left(e^{\tau+s}-\left(1-\delta_{1}\right)\right) e^{\tau+s} u_{0}\left(e^{\tau+s}-\left(1-\delta_{1}\right)\right)\right|^{2} d s \lesssim \int_{0}^{\infty}\left|u_{0}(r)\right|^{2} r^{3} d r \lesssim 1, \\
\int_{s_{0}}\left|\left(e^{\tau+s}-\left(1-\delta_{1}\right)\right)^{2} e^{\tau+s} u_{0}^{\prime}\left(e^{\tau+s}-\left(1-\delta_{1}\right)\right)\right|^{2} d s \lesssim \int_{0}^{\infty}\left|\partial_{r} u_{0}(r)\right|^{2} r^{5} d r \lesssim 1 .
\end{gathered}
$$

By the radial Sobolev inequality (2.2), we have $\left|u_{0}(r)\right| \lesssim r^{-2}$. This estimate and the inequality (2.3) imply

$$
\begin{aligned}
& \int_{s_{0}}^{\infty}\left|\left(1-\delta_{1}-e^{\tau-s}\right) e^{\tau-s} u_{0}\left(1-\delta_{1}-e^{\tau-s}\right)\right|^{2} d s+\int_{s_{0}}^{\infty} \mid\left(\left.\left(1-\delta_{1}-e^{\tau-s}\right)^{2} u_{0}^{\prime}\left(1-\delta_{1}-e^{\tau-s}\right) e^{\tau-s}\right|^{2} d s\right. \\
\lesssim & \int_{s_{0}}^{\infty} e^{-2 s} d s \lesssim 1 .
\end{aligned}
$$

We now take the derivatives of (5.19) with respect to $\tau$ and $s$, then

$$
\partial_{\tau}(\underline{5.19})=\frac{1-\delta_{1}}{2 e^{\tau} \sinh s} \int_{1-\delta_{1}-e^{\tau-s}}^{e^{\tau+s}-\left(1-\delta_{1}\right)} \rho u_{0}(\rho) d \rho+I_{1}+I_{2}
$$

and

where

$$
\partial_{s}(\underline{5.19})=\partial_{s}\left(\frac{e^{\tau} \cosh s-\left(1-\delta_{1}\right)}{2 e^{\tau} \sinh s}\right) \int_{1-\delta_{1}-e^{\tau-s}}^{e^{\tau+s}-\left(1-\delta_{1}\right)} \rho u_{0}(\rho) d \rho+I_{1}-I_{2}
$$

$$
\begin{gathered}
I_{1}=\frac{e^{\tau} \cosh s-\left(1-\delta_{1}\right)}{2 e^{\tau} \sinh s} e^{\tau+s}\left(e^{\tau+s}-\left(1-\delta_{1}\right)\right) u_{0}\left(e^{\tau+s}-\left(1-\delta_{1}\right)\right), \\
I_{2}=\frac{e^{\tau} \cosh s-\left(1-\delta_{1}\right)}{2 e^{\tau} \sinh s} e^{\tau-s}\left(1-\delta_{1}-e^{\tau-s}\right) u_{0}\left(1-\delta_{1}-e^{\tau-s}\right) .
\end{gathered}
$$

For the first term in the right hand of (5.27), by the inequality (2.3), we have

$$
\int_{s_{0}}^{\infty}\left|e^{-s} \int_{1-\delta_{1}-e^{\tau-s}}^{e^{\tau+s}-\left(1-\delta_{1}\right)} \rho u_{0}(\rho) d \rho\right|^{2} d s \lesssim \int_{s_{0}}^{\infty} e^{-2 s} d s \lesssim 1
$$


By similar estimates, one can find that the contribution of the first term in the right hand of (5.28) to (5.16) is finite. For $I_{1}$, changing of variables and the inequality (2.13) yield

$$
\int_{s_{0}}^{\infty}\left|I_{1}\right|^{2} d s \lesssim \int_{s_{0}}^{\infty}\left|e^{2 s} u_{0}\left(e^{\tau+s}-\left(1-\delta_{1}\right)\right)\right|^{2} d s \lesssim \int_{\frac{1}{2} e^{s_{0}}}^{\infty} \rho^{3}\left|u_{0}(\rho)\right|^{2} d \rho \lesssim 1
$$

For $I_{2}$, by (2.2), we obtain

$$
\int_{s_{0}}^{\infty}\left|I_{2}\right| d s \lesssim \int_{s_{0}}^{\infty}\left|e^{-s}\right|^{2} d s \lesssim 1
$$

Next, we consider the contribution of (5.20) to (5.16). For simplicity, we consider

$$
\begin{aligned}
\frac{1}{2}\left(\partial_{\tau}-\partial_{s}\right)(\underline{(5.20)})= & e^{\tau-s}\left(1-\delta_{1}-e^{\tau-s}\right)^{2} u_{1}\left(1-\delta_{1}-e^{\tau-s}\right) \\
& +\frac{e^{\tau-s}\left(e^{\tau+s}-\left(1-\delta_{1}\right)\right)^{2}}{\left(e^{\tau+s}-e^{\tau-s}\right)^{2}} \int_{1-\delta_{1}-e^{\tau-s}}^{e^{\tau+s}-\left(1-\delta_{1}\right)} \rho u_{1}(\rho) d \rho \\
& +\frac{e^{\tau-s}}{\left(e^{\tau+s}-e^{\tau-s}\right)^{2}} \int_{1-\delta_{1}-e^{\tau-s}}^{e^{\tau+s}-\left(1-\delta_{1}\right)} \rho^{3} u_{1}(\rho) d \rho,
\end{aligned}
$$

and

$$
\begin{aligned}
\frac{1}{2}\left(\partial_{\tau}+\partial_{s}\right)(\underline{5.20})= & e^{\tau+s}\left(e^{\tau+s}-\left(1-\delta_{1}\right)\right)^{2} u_{1}\left(e^{\tau+s}-\left(1-\delta_{1}\right)\right) \\
& +\frac{e^{\tau+s}\left(1-\delta_{1}-e^{\tau-s}\right)^{2}}{\left(e^{\tau+s}-e^{\tau-s}\right)^{2}} \int_{1-\delta_{1}-e^{\tau-s}}^{e^{\tau+s}-\left(1-\delta_{1}\right)} \rho u_{1}(\rho) d \rho \\
& +\frac{e^{\tau+s}}{\left(e^{\tau+s}-e^{\tau-s}\right)^{2}} \int_{1-\delta_{1}-e^{\tau-s}}^{e^{\tau+s}-\left(1-\delta_{1}\right)} \rho^{3} u_{1}(\rho) d \rho .
\end{aligned}
$$

Using the estimates (2.4) and (2.13), we can easily estimate the contributions of (5.34)-(5.38) to (5.16). Let $\mathbb{I}_{J}(y)$ be the characteristic function of an interval $J \subset \mathbb{R}$. For (5.39), by the inequality (2.13) and a change of variables, we see that

$$
\begin{aligned}
\int_{s_{0}}^{\infty}\left|e^{-s} \int_{1-\delta_{1}-e^{\tau-s}}^{e^{\tau+s}-\left(1-\delta_{1}\right)} \rho^{3} u_{1}(\rho) d \rho\right|^{2} d s & \lesssim \int_{s_{0}}^{\infty}\left|e^{-s} \int_{0}^{2 e^{s}} \rho^{3}\right| u_{1}(\rho)|d \rho|^{2} d s \\
& \lesssim \int_{0}^{\infty}\left|\frac{1}{\eta} \int_{0}^{2 \eta} \rho^{3}\right| u_{1}(\rho)|d \rho|^{2} \frac{1}{\eta} d \eta \\
& \lesssim \int_{0}^{\infty}\left|\frac{1}{\eta} \int_{0}^{2 \eta} \rho^{\frac{5}{2}}\right| u_{1}(\rho)|d \rho|^{2} d \eta \\
& \lesssim \int_{0}^{\infty}\left|\mathcal{M}\left(\mathbb{I}_{[0, \infty)}(\rho) \rho^{\frac{5}{2}} u_{1}(\rho)\right)\right|^{2}(\eta) d \eta \\
& \lesssim \int_{0}^{\infty}\left|u_{1}(\rho)\right|^{2} \rho^{5} d \rho \lesssim 1,
\end{aligned}
$$

where $\mathcal{M}$ is the Hardy-Littlewood maximal function and we used the fact that $\mathcal{M}$ is bounded in $L^{2}$. 
Next, we consider the contribution of (5.21) to the energy (5.16). Also, for simplicity, we consider

$$
\begin{aligned}
\left(\partial_{\tau}+\partial_{s}\right)(\underline{5.21})= & e^{\tau+s} \int_{1-\delta_{1}}^{e^{\tau} \cosh s}\left(e^{\tau+s}-t\right)^{2}(|\bar{u}| \bar{u})\left(t, e^{\tau+s}-t\right) d t \\
& +\frac{e^{\tau+s}}{\left(e^{\tau+s}-e^{\tau-s}\right)^{2}} \int_{1-\delta_{1}}^{e^{\tau} \cosh s} \int_{t-e^{\tau-s}}^{e^{\tau+s}-t} \rho\left(t-e^{\tau-s}\right)^{2}(|\bar{u}| \bar{u})(t, \rho) d \rho d t \\
& -\frac{e^{\tau+s}}{\left(e^{\tau+s}-e^{\tau-s}\right)^{2}} \int_{1-\delta_{1}}^{e^{\tau} \cosh s} \int_{t-e^{\tau-s}}^{e^{\tau+s}-t} \rho^{3}(|\bar{u}| \bar{u})(t, \rho) d \rho d t
\end{aligned}
$$

and

$$
\begin{aligned}
\left(\partial_{\tau}-\partial_{s}\right) \underline{(5.21)}= & e^{\tau-s} \int_{1-\delta_{1}}^{e^{\tau} \cosh s}\left(t-e^{\tau-s}\right)^{2}|\bar{u}| \bar{u}\left(t, t-e^{\tau-s}\right) d t \\
& -\frac{e^{\tau-s}}{\left(e^{\tau+s}-e^{\tau-s}\right)^{2}} \int_{1-\delta_{1}}^{e^{\tau} \cosh s} \int_{t-e^{\tau-s}}^{e^{\tau+s}-t} \rho\left(e^{\tau+s}-t\right)^{2}|\bar{u}| \bar{u}(t, \rho) d \rho d t \\
& +\frac{e^{\tau-s}}{\left(e^{\tau+s}-e^{\tau-s}\right)^{2}} \int_{1-\delta_{1}}^{e^{\tau} \cosh s} \int_{t-e^{\tau-s}}^{e^{\tau+s}-t} \rho^{3}|\bar{u}| \bar{u}(t, \rho) d \rho d t
\end{aligned}
$$

By the definition of $\bar{u}$, the inequality (3.23) and Hölder, the contribution of (5.41) can be estimated as

$$
\begin{aligned}
& \left.\left.\int_{s_{0}}^{\infty}\left|e^{\tau+s} \int_{1-\delta_{1}}^{e^{\tau} \cosh s}\left(e^{\tau+s}-t\right)^{2}\right| \bar{u}\right|^{2}\left(t, e^{\tau+s}-t\right) d t\right|^{2} d s \\
\lesssim & \int_{s_{0}}^{\infty} \int_{1-\delta_{1}}^{e^{\tau} \cosh s}|\bar{u}|^{4}\left(t, e^{\tau+s}-t\right) e^{7 s} d t d s \\
\lesssim & \int_{\frac{1}{4} e^{s_{0}}}^{\infty} \int_{1-\delta_{1}}^{\rho}|\bar{u}|^{4}(t, \rho) \rho^{6} d t d \rho \\
\lesssim & \int_{0}^{\infty} \int_{\rho>t+\frac{1}{2}}|u|^{4}(t, \rho) \rho^{6} d \rho d t \\
\lesssim & \|u\|_{L^{3}\left(\left\{|x|>|t|+\frac{1}{2}\right\}\right)}^{3} \sup _{t \geq 0}\left\||x|^{2} u(t, x)\right\|_{L_{x}^{\infty}\left(\left\{|x|>t+\frac{1}{2}\right\}\right)} \lesssim 1 .
\end{aligned}
$$


Now, we consider (5.42) and (5.43). By Hölder, a change of variables, and the inequality (3.23), we have

$$
\begin{aligned}
& \left.\left.\int_{s_{0}}^{\infty}\left|\int_{1-\delta_{1}}^{e^{\tau} \cosh s} e^{-s} \int_{t-e^{\tau-s}}^{e^{\tau+s}-t}\left(t^{2} \rho+\rho^{3}\right)\right| \bar{u}\right|^{2}(t, \rho) d \rho d t\right|^{2} d s \\
\lesssim & \int_{s_{0}}^{\infty}\left|\int_{1-\delta_{1}}^{e^{\tau} \cosh s} \mathcal{M}\left(\mathbb{I}_{\left[t-\frac{1}{2}+\delta_{1}, \infty\right)}(\rho) \rho^{3}|\bar{u}|^{2}(t, \rho)\right)\left(e^{\tau} \sinh s\right) d t\right|^{2} d s \\
\lesssim & \int_{s_{0}}^{\infty} \int_{1-\delta_{1}}^{e^{\tau} \cosh s}\left[\mathcal{M}\left(\mathbb{I}_{\left[t-\frac{1}{2}+\delta_{1}, \infty\right)}(\rho) \rho^{3}|\bar{u}|^{2}(t, \rho)\right)\left(e^{\tau} \sinh s\right)\right]^{2} e^{\tau} \cosh s d t d s \\
\lesssim & \int_{1-\delta_{1}}^{\infty} \int_{e^{s_{0}}}^{\infty}\left[\mathcal{M}\left(\mathbb{I}_{\left[t-\frac{1}{2}+\delta_{1}, \infty\right)}(\rho) \rho^{3}|\bar{u}|^{2}(t, \rho)\right)(r)\right]^{2} d r d t \\
\lesssim & \int_{1-\delta_{1}}^{\infty} \int_{r>t-\frac{1}{2}+\delta_{1}} r^{6}|\bar{u}|^{4}(t, r) d r d t \\
\lesssim & \int_{0}^{\infty} \int_{\rho>t+\frac{1}{2}}|u|^{4}(t, \rho) \rho^{6} d \rho d t \\
\lesssim & \|u\|_{L^{3}\left(\left\{|x|>|t|+\frac{1}{2}\right\}\right)}^{3} \sup _{t \geq 0}\left\||x|^{2} u(t, x)\right\|_{L_{x}^{\infty}\left(\left\{|x|>t+\frac{1}{2}\right\}\right)} \lesssim 1 .
\end{aligned}
$$

Thus, the contribution of (5.42) and (5.43) to (5.16) is finite.

For (5.44), by the fact that $e^{\tau-s_{0}}<\frac{1}{2}-\delta_{1}$, the definition of $\bar{u}$, and the inequality (3.23), we have

$$
\begin{aligned}
& \left.\left.\int_{s_{0}}^{\infty} e^{-2 s}\left|\int_{1-\delta_{1}}^{e^{\tau} \cosh s}\left(t-e^{\tau-s}\right)^{2}\right| \bar{u}\right|^{2}\left(t, t-e^{\tau-s}\right) d t\right|^{2} d s \\
\lesssim & \int_{s_{0}}^{\infty} e^{-2 s}\left|\int_{1-\delta_{1}}^{e^{\tau} \cosh s} t^{-2} d t\right|^{2} \lesssim 1 .
\end{aligned}
$$

Similarly, for (5.45) and (5.46), by the fact that $e^{\tau-s_{0}}<\frac{1}{2}-\delta_{1}$ and the inequality (3.23), we can obtain that

$$
\begin{aligned}
& \left.\left.\int_{s_{0}}^{\infty} e^{-6 s}\left|\int_{1-\delta_{1}}^{e^{\tau} \cosh s} \int_{t-e^{\tau-s}}^{e^{\tau+s}-t}\left[\rho^{3}+\rho\left(e^{\tau+s}-t\right)^{2}\right]\right| \bar{u}\right|^{2}(t, \rho) d \rho d t\right|^{2} d s \\
\lesssim & \left.\left.\int_{s_{0}}^{\infty} e^{-2 s}\left|\int_{1-\delta_{1}}^{e^{\tau} \cosh s} \int_{t-e^{\tau-s}}^{e^{\tau+s}-t} \rho\right| \bar{u}\right|^{2}(t, \rho) d \rho d t\right|^{2} d s \\
\lesssim & \int_{s_{0}}^{\infty} e^{-2 s}\left|\int_{1-\delta_{1}}^{e^{\tau} \cosh s} \int_{t-e^{\tau-s}}^{e^{\tau+s}-t} \rho^{-3} d \rho d t\right|^{2} d s \\
\lesssim & \int_{s_{0}}^{\infty} e^{-2 s}\left|\int_{1-\delta_{1}}^{e^{\tau} \cosh s} t^{-2} d t\right|^{2} d s \lesssim 1 .
\end{aligned}
$$

Hence, combining (5.24)-(5.50), we have

$$
\int_{s_{0}}^{\infty} \frac{1}{2}\left|\left(s^{2} \tilde{w}\right)_{\tau}\right|^{2}+\frac{1}{2}\left|\left(s^{2} \tilde{w}\right)_{s}\right|^{2} d s \lesssim 1
$$


5.3.2. The hyperbolic energy for $0 \leq s \leq s_{0}$. By the hyperbolic transform (5.5), we have

$$
\begin{aligned}
\left(s^{2} \tilde{w}\right)_{\tau}(\tau, s)= & 2 e^{2 \tau} \sinh ^{2} s \bar{w}\left(e^{\tau} \cosh s, e^{\tau} \sinh s\right)+e^{3 \tau} \sinh ^{2} s \cosh s \bar{w}_{t}\left(e^{\tau} \cosh s, e^{\tau} \sinh s\right) \\
& +e^{3 \tau} \sinh ^{3} s \bar{w}_{r}\left(e^{\tau} \cosh s, e^{\tau} \sinh s\right) \\
\left(s^{2} \tilde{w}\right)_{s}(\tau, s)= & 2 e^{2 \tau} \sinh s \cosh s \bar{w}\left(e^{\tau} \cosh s, e^{\tau} \sinh s\right)+e^{3 \tau} \sinh ^{3} s \bar{w}_{t}\left(e^{\tau} \cosh s, e^{\tau} \sinh s\right) \\
& +e^{3 \tau} \sinh ^{2} s \cosh s \bar{w}_{r}\left(e^{\tau} \cosh s, e^{\tau} \sinh s\right) .
\end{aligned}
$$

Hence

$$
\begin{aligned}
& \int_{0}^{1} \int_{0}^{s_{0}}\left|\left(s^{2} \tilde{w}\right)_{\tau}\right|^{2}+\left|\left(s^{2} \tilde{w}\right)_{s}\right|^{2} d s d \tau \\
\lesssim & \int_{0}^{1} \int_{0}^{s_{0}} e^{4 \tau} \sinh ^{2} s\left(\sinh ^{2} s+\cosh ^{2} s\right)|\bar{w}|^{2}\left(e^{\tau} \cosh s, e^{\tau} \sinh s\right) d s d \tau \\
& +\int_{0}^{1} \int_{0}^{s_{0}} e^{6 \tau} \sinh ^{4} s\left(\sinh ^{2} s+\cosh ^{2} s\right)\left[\bar{w}_{t}^{2}+\bar{w}_{r}^{2}\right]\left(e^{\tau} \cosh s, e^{\tau} \sinh s\right) d s d \tau .
\end{aligned}
$$

Taking $C_{0}=e^{1+s_{0}}$, by a change of variables, the Hardy inequality and the inequality (3.68), we obtain

$$
(\underline{5.54}) \lesssim \iint_{|x|+|t| \leq C_{0}} \frac{1}{|x|^{2}}|\bar{w}|^{2}(t, x) d x d t \lesssim \sup _{0<t<C_{0}}\left\|\nabla_{x} \bar{w}\right\|_{L_{x}^{2}\left(\mathbb{R}^{5}\right)} \lesssim 1 .
$$

Similarly, for (5.55), changing of variables, we have

$$
\left(\underline{5.55)} \lesssim \iint_{|x|+|t| \leq C_{0}}\left|\nabla_{t, x} \bar{w}\right|^{2}(t, x) d x d t \lesssim \sup _{0<t<C_{0}}\left\|\nabla_{t, x} \bar{w}\right\|_{L_{x}^{2}\left(\mathbb{R}^{5}\right)} \lesssim 1\right.
$$

Then, by the mean value theorem, there exists $\tau_{0} \in[0,1]$, such that

$$
\int_{0}^{s_{0}}\left|\left(s^{2} \tilde{w}\right)_{\tau}\right|^{2}\left(\tau_{0}, s\right)+\left|\left(s^{2} \tilde{w}\right)_{s}\right|^{2}\left(\tau_{0}, s\right) d s \lesssim 1
$$

This estimate along with (5.51) implies

$$
\int_{0}^{\infty}\left|\left(s^{2} \tilde{w}\right)_{\tau}\right|^{2}\left(\tau_{0}, s\right)+\left|\left(s^{2} \tilde{w}\right)_{s}\right|^{2}\left(\tau_{0}, s\right) d s \lesssim 1
$$

5.4. Uniform boundedness of the hyperbolic energy of $\tilde{w}$. We are now going to show that $E_{h}(\tilde{w})(\tau)$ is uniformly bounded for $\tau \in \mathbb{R}_{+}$.

A simple calculation gives

$$
\frac{d}{d \tau} E_{h}(\tilde{w}(\tau))=\int \frac{\left|s^{2} \tilde{w}\right| s^{2} \tilde{w}-\left|s^{2} \tilde{u}\right| s^{2} \tilde{u}}{\sinh ^{2} s} s^{2} \tilde{w}_{\tau} d s .
$$

Utilizing the decay property (3.37) of $v$, we have, for $\tau, s \geq 0$,

$$
\left(e^{\tau} \cosh s-\left(1-\delta_{1}\right)\right)^{2} v\left(e^{\tau} \cosh s-\left(1-\delta_{1}\right), e^{\tau} \sinh s\right) \lesssim \delta_{1}^{-\frac{1}{2}} .
$$

The Huygens principle implies that $v\left(e^{\tau} \cosh s-\left(1-\delta_{1}\right), e^{\tau} \sinh s\right)=0$ unless $1-\frac{6}{5} \delta_{1} \leq e^{\tau-s} \leq 1-\frac{4}{5} \delta_{1}$. Thus, for $\tau, s \geq 0$, we have

$$
\frac{s^{2}|\tilde{v}(\tau, s)|}{\sinh ^{2} s}=e^{2 \tau}\left|v\left(e^{\tau} \cosh s-\left(1-\delta_{1}\right), e^{\tau} \sinh s\right)\right| \lesssim \frac{e^{2 \tau} \mathbb{I}_{\left\{s \geq 0: e^{\tau-s} \leq 1-\frac{4}{5} \delta_{1}\right\}}}{\left(e^{\tau} \cosh s-\left(1-\delta_{1}\right)\right)^{2}} \lesssim e^{-\tau} .
$$


Hence, by Hölder and interpolation, we have

$$
\begin{aligned}
\int_{0}^{\infty} \frac{1}{\sinh ^{2} s}\left|s^{2} \tilde{w}_{\tau}\right|\left|s^{2} \tilde{v}\right|^{2} d s & \lesssim\left(\int_{0}^{\infty}\left|s^{2} \tilde{w}_{\tau}\right|^{2} d s\right)^{\frac{1}{2}}\left(\int_{0}^{\infty} \frac{1}{\sinh ^{2} s}\left|s^{2} \tilde{v}\right|^{3} d s\right)^{\frac{1}{2}}\left\|\frac{s^{2}}{\sinh ^{2} s} \tilde{v}(\tau, s)\right\|_{L_{s}^{\infty}}^{\frac{1}{2}} \\
\lesssim & e^{-\tau / 2} E_{h}(\tilde{w}(\tau))^{\frac{1}{2}}\left(\int_{0}^{\infty} \frac{1}{\sinh ^{2} s}\left|s^{2} \tilde{v}\right|^{3} d s\right)^{\frac{1}{2}} \\
\int_{0}^{\infty} \frac{1}{\sinh ^{2} s}\left|s^{2} \tilde{w}_{\tau}\right|\left|s^{2} \tilde{v}\right|\left|s^{2} \tilde{w}\right| d s & \lesssim\left(\int_{0}^{\infty}\left|s^{2} \tilde{w}_{\tau}\right|^{2} d s\right)^{\frac{1}{2}}\left(\int_{0}^{\infty} \frac{1}{\sinh ^{2} s}\left|s^{2} \tilde{w}\right|^{3} d s\right)^{\frac{1}{3}}\left(\int_{0}^{\infty}\left|s^{2} \tilde{v}(\tau, s)\right|^{6} \frac{1}{\sinh ^{8} s} d s\right)^{\frac{1}{6}} \\
& \lesssim e^{-\tau / 2} E_{h}(\tilde{w}(\tau))^{\frac{5}{6}}\left(\int_{0}^{\infty} \frac{1}{\sinh ^{2} s}\left|s^{2} \tilde{v}\right|^{3} d s\right)^{\frac{1}{6}} .
\end{aligned}
$$

Combining (5.63) with (5.64) and employing Hölder again, we have

$$
\frac{d}{d \tau} E_{h}\left(\tilde { w } ( \tau ) \lesssim e ^ { - \tau / 2 } \left[E_{h}\left(\tilde{w}(\tau)+\int_{0}^{\infty} \frac{1}{\sinh ^{2} s}\left|s^{2} \tilde{v}\right|^{3} d s\right]\right.\right.
$$

On the other hand, changing of variables, we have

$$
\int_{0}^{\infty} \int_{0}^{\infty} \frac{1}{\sinh ^{2} s}\left|s^{2} \tilde{v}\right|^{3} d s d \tau \leq \int_{\delta_{1}}^{\infty} \int_{0}^{\infty}|v(t, r)|^{3} r^{4} d r d t \leq\|v\|_{L_{t, x}^{3}\left(\left[\delta_{1}, \infty\right) \times \mathbb{R}^{5}\right)}^{3} \lesssim 1 .
$$

Hence, by Gronwall's inequality, (5.65) and (5.66) yield that $E_{h}(\tilde{w})(\tau)$ is uniformly bounded in $\mathbb{R}_{+}$.

5.5. Concluding of the proof of Proposition 1.2. We complete the proof of Proposition 1.2 by studying the Morawetz action in hyperbolic coordinates.

Proposition 5.1. Let $\tilde{w}$ be defined in (5.5), then

$$
\iint_{\Omega_{2}}|w(t, r)|^{3} r^{4} d t d r=\int_{0}^{\infty} \int_{0}^{\infty} \frac{\left|s^{2} \tilde{w}\right|^{3}}{\sinh ^{2} s} d s d \tau \lesssim 1
$$

Proof. Define the Morawetz action by

$$
M(\tau)=\int_{0}^{\infty}\left(s^{2} \tilde{w}\right)_{\tau}\left(s^{2} \tilde{w}\right)_{s} d s=\int_{0}^{\infty} \tilde{w}_{\tau}\left(\tilde{w}_{s}+\frac{2}{s} \tilde{w}\right) s^{4} d s
$$

One can easily find that $|M(\tau)| \leq E_{h}(\tilde{w})(\tau)$. By (5.8), we have

$$
\begin{aligned}
\frac{d}{d \tau} M(\tau)= & -\int_{0}^{\infty} \frac{2 s^{2} \tilde{w}}{\sinh ^{2} s}\left(s^{2} \tilde{w}\right)_{s} d s-\int_{0}^{\infty} \frac{s^{4}}{\sinh ^{2} s}|\tilde{u}| \tilde{u} s^{2} \tilde{w}_{s} d s \\
= & -2 \int_{0}^{\infty} \frac{\left|s^{2} \tilde{w}\right|^{2}}{\sinh ^{2} s} \frac{\cosh s}{\sinh s} d s-\frac{2}{3} \int_{0}^{\infty} \frac{\left|s^{2} \tilde{w}\right|^{3}}{\sinh ^{2} s} \frac{\cosh s}{\sinh s} d s \\
& +\int_{0}^{\infty} \frac{s^{4}}{\sinh ^{2} s}(|\tilde{w}| \tilde{w}-|\tilde{u}| \tilde{u}) s^{2} \tilde{w}_{s} d s .
\end{aligned}
$$


By Hölder, the estimate (5.62), and the fact that $E_{h}(\tilde{w}(\tau))$ is uniformly bounded for $\tau \geq 0$, we have

$$
\begin{aligned}
& \left|\int_{0}^{\infty} \int_{0}^{\infty} \frac{s^{4}}{\sinh ^{2} s}(|\tilde{w}| \tilde{w}-|\tilde{u}| \tilde{u}) s^{2} \tilde{w}_{s} d s d \tau\right| \\
\lesssim & \int_{0}^{\infty} \int_{0}^{\infty} \frac{1}{\sinh ^{2} s}\left|s^{2} \tilde{w}_{\tau}\right|\left|s^{2} \tilde{v}\right|^{2} d s d \tau+\int_{0}^{\infty} \int_{0}^{\infty} \frac{1}{\sinh ^{2} s}\left|s^{2} \tilde{w}_{\tau}\right|\left|s^{2} \tilde{v} \| s^{2} \tilde{w}\right| d s d \tau \\
\lesssim & \int_{0}^{\infty} e^{-\tau} E_{h}(\tilde{w}(\tau))^{\frac{1}{2}}\left(\int_{0}^{\infty} \frac{1}{\sinh ^{2} s}\left|s^{2} \tilde{v}\right|^{3} d s\right)^{\frac{1}{2}} \tau \\
& +\int_{0}^{\infty} e^{-\tau} E_{h}(\tilde{w}(\tau))^{\frac{5}{6}}\left(\int_{0}^{\infty} \frac{1}{\sinh ^{2} s}\left|s^{2} \tilde{v}\right|^{3} d s\right)^{\frac{1}{6}} d \tau \\
\lesssim & \|v\|_{L_{t, x}^{3}\left(\mathbb{R} \times \mathbb{R}^{5}\right)}^{3} 1 .
\end{aligned}
$$

This together with the equality (5.69) and the fact $M(\tau)$ is uniformly bounded for $\tau \geq 0$, implies that

$$
\int_{0}^{\infty} \int_{0}^{\infty} \frac{\left|s^{2} \tilde{w}\right|^{3}}{\sinh ^{2} s} \frac{\cosh s}{\sinh s} d s d \tau \lesssim 1
$$

Thus, we have

$$
\int_{0}^{\infty} \int_{0}^{\infty} \frac{\left|s^{2} \tilde{w}\right|^{3}}{\sinh ^{2} s} d s d \tau \lesssim 1
$$

This yields (5.67) by the definition of $\tilde{w}$.

Acknowledgments: This work is supported in part by the National Natural Science Foundation of China under grant No.11671047.

\section{REFERENCES}

[1] Hajer Bahouri and Patrick Gérard. High frequency approximation of solutions to critical nonlinear wave equations. American Journal of Mathematics, 121(1):131-175, 1999.

[2] Hajer Bahouri and Jalal Shatah. Decay estimates for the critical semilinear wave equation. Annales De L Institut Henri Poincare-analyse Non Lineaire, 15(6):783-789, 1998.

[3] Rodriguez Casey. Scattering for radial energy-subcritical wave equations. arXiv:1602.04540.

[4] F. M. Christ and Michael. I. Weinstein. Dispersion of small amplitude solutions of the generalized Korteweg-de Vries equation. Journal of Functional Analysis, 100(1):87 - 109, 1991.

[5] Leonardo Colzani, Alice Cominardi, and Krzysztof Stempak. Radial solutions to the wave equation. Annali Di Matematica Pura Ed Applicata, 181(1):25-54, 2002.

[6] Benjamin Dodson. Global well-posedness and scattering for the radial, defocusing, cubic wave equation with initial data in a critical Besov space. arXiv:1608.02020v1.

[7] Jean Ginibre and Giorgio Velo. The global Cauchy problem for the nonlinear Klein-Gordon equation. Mathematische Zeitschrift, 189(4):487-505, 1985.

[8] Jean Ginibre and Giorgio Velo. The global Cauchy problem for the non linear Klein-Gordon equation-II. In Annales de l'Institut Henri Poincare (C) Non Linear Analysis, volume 6, pages 15-35. Elsevier, 1989.

[9] Jean Ginibre and Giorgio Velo. Generalized Strichartz Inequalities for the Wave Equation. Journal of Functional Analysis, 133(1):153-160, 1995.

[10] Manoussos G Grillakis. Regularity and asymptotic behavior of the wave equation with a critical nonlinearity. Annals of Mathematics, 132(3):485-509, 1990.

[11] Manoussos G Grillakis. Regularity for the wave equation with a critical nonlinearity. Communications on Pure and Applied Mathematics, 45(6):749-774, 1992.

[12] Markus Keel and Terence Tao. Endpoint Strichartz estimates. American Journal of Mathematics, 120(5):955-980, 1998.

[13] Carlos Kenig. Lectures on the Energy Critical Nonlinear Wave Equation, volume 122. American Mathematical Society, 2015.

[14] Carlos E. Kenig and Frank Merle. Global well-posedness, scattering and blow-up for the energy-critical, focusing, non-linear Schrödinger equation in the radial case. Inventiones mathematicae, 166(3):645-675, Dec 2006.

[15] Carlos E Kenig and Frank Merle. Global well-posedness, scattering and blow-up for the energy-critical focusing non-linear wave equation. Acta Mathematica, 201(2):147-212, 2008. 
[16] Rowan Killip and Monica Visan. Nonlinear Schrödinger equations at critical regularity, 2009.

[17] Rowan Killip and Monica Visan. The defocusing energy-supercritical nonlinear wave equation in three space dimensions. Transactions of the American Mathematical Society, 363(7):3893-3934, 2011.

[18] Herbert Koch, Daniel Tataru, and Monica Visan. Dispersive Equations and Nonlinear Waves. Springer Basel, 2014.

[19] Hans Lindblad and Christopher D Sogge. On existence and scattering with minimal regularity for semilinear wave equations. Journal of Functional Analysis, 130(2):357-426, 1995.

[20] Hans Lindblad and Christopher D Sogge. Long-time existence for small amplitude semilinear wave equations. American Journal of Mathematics, 118(5):1047-1135, 1996.

[21] Cathleen S Morawetz. Time decay for the nonlinear Klein-Gordon equation. In Proceedings of the Royal Society of London A: Mathematical, Physical and Engineering Sciences, volume 306, pages 291-296. The Royal Society, 1968.

[22] Kenji Nakanishi. Unique global existence and asymptotic behaviour of solutions for wave equations with non-coercive critical nonlinearity. Communications in Partial Differential Equations, 24:185-221, 1999.

[23] Mohammad A Rammaha. Finite-time blow-up for nonlinear wave equations in high dimensions. Communications in Partial Differential Equations, 12(6):677-700, 1987.

[24] Javier Ramos. A refinement of the Strichartz inequality for the wave equation with applications. Advances in Mathematics, 230(2):649-698, 2012.

[25] Jalal Shatah and Michael Struwe. Regularity results for nonlinear wave equations. Annals of Mathematics, 138(3):503-518, 1993.

[26] Christopher D Sogge. Lectures on non-linear wave equations, volume 2. International Press Boston, MA, 1995.

[27] Robert Taggart. Inhomogeneous Strichartz estimates. Forum Mathematicum, 22(5):825-853, 2008.

Changxing Miao:

Institute of Applied Physics and Computational Mathematics,

P. O. Box 8009, BeiJing, China, 100088,

E-mail address: miao_changxing@iapcm.ac.cn

JIANWEI YANG:

Beijing International Center for Mathematical Research, Peking University, Beijing 100871, China

E-mail address: geewey_young@pku.edu.cn

Tengfei ZhaO:

The Graduate School of China Academy of Engineering Physics,

P. O. Box 2101, Beijing, China, 100088,

E-mail address: zhao_tengfei@yeah.net 